\title{
TWIN-CONVERGENCE REGIONS FOR CONTINUED FRACTIONS $K\left(a_{n} / 1\right)$
}

\author{
BY \\ WILLIAM B. JONES( $\left.{ }^{1}\right)$ AND W. J. THRON
}

1. Introduction. A pair of nonempty sets $\left(E_{1}, E_{2}\right)$ will be called twin-convergence regions for continued fractions

$$
K_{n=1}^{\infty}\left(a_{n} / 1\right)=\frac{a_{1}}{1}+\frac{a_{2}}{1}+\frac{a_{3}}{1}+\cdots
$$

if the conditions

$$
a_{2 n-1} \in E_{1}, \quad a_{2 n} \in E_{2}, \quad a_{n} \neq 0, \quad n=1,2,3, \ldots,
$$

insure the convergence of (1.1).

The first significant twin-convergence region result is due to Leighton and Wall [12] who proved in 1936 that $\left|a_{2 n}\right| \leqq 1 / 4,\left|a_{2 n-1}\right| \geqq 25 / 4$ is sufficient for the convergence of (1.1). Since then a great many other twin-convergence regions have been obtained [2], [3], [4], [11], [14], [15], [17]. When we refer to earlier results here we occasionally state them in a form different from but equivalent to that given originally. The best result known so far is the theorem of Lange and Thron [9], [10] which states that if we set $a_{n}=c_{n}^{2}$ then the conditions

$$
\begin{array}{rlrl}
\left|c_{2 n}+i \Gamma\right| & \leqq \rho, & \left|c_{2 n}-i \Gamma\right| & \leqq \rho, \\
\left|c_{2 n-1}+i(i+\Gamma)\right| & \geqq \rho, \quad\left|c_{2 n-1}-i(1+\Gamma)\right| & \geqq \rho,
\end{array}
$$

where $\Gamma$ is a complex number and $|\Gamma|<\rho<|1+\Gamma|$, are sufficient for the convergence of (1.1). A result of a different type originally due to Cowling, Leighton and Thron [3] and later improved by one of the authors [17] gives

$$
\left|a_{2 n}\right| \leqq \rho, \quad\left|a_{2 n-1}\right| \geqq 2\left(\rho-\cos \arg a_{2 n-1}\right), \quad p>1,
$$

as twin-convergence regions.

In the present paper we are first of all concerned with the exposition of a new method which yields a very large number of twin-convergence regions, including most of the known results. The number is so large that we must try to come to grips with the problem of eliminating redundancies. One means of accomplishing this is by developing necessary conditions, in terms of value regions, for a pair of

Received by the editors June 12, 1969 and, in revised form, October 20, 1969.

(1) Supported in part by the National Science Foundation under Grant No. GP-9009.

Copyright (C) 1970, American Mathematical Society 
regions to be twin-convergence regions. This is done in $\S 3$, using ideas originally employed by one of the authors [16] to derive necessary conditions for simpleconvergence regions. It may be of interest to note that Theorem 3.1, which plays a key role in this context, cannot be extended to $n$-tuple-convergence regions. This may be a reason why so much more is known about $n$-tuple-convergence regions when $n=1,2$ than when $n>2$.

We return to the problem of eliminating redundancies in $\S 6$. There we assume that the value regions under consideration satisfy

$$
-1 \notin V_{1} \cup V_{2} \text { and } \quad V_{1} \cap\left(-1-V_{2}\right)=\varnothing
$$

and we make the conjecture that all such regions can be eliminated except those for which $V_{1}=-1-C\left(V_{2}\right)$. We are able to prove the conjecture in some cases.

In view of what has been said above, it must be pointed out that there may still be some overlap in the convergence results contained in this article. However, it is shown in $\S 5$ that the theorem of Lange and Thron is a special case of Theorem 5.4. Furthermore, the results obtained from Theorem 5.4 for

$$
\left|\Gamma_{1}\right|<\rho_{1}, \quad\left|1+\Gamma_{1}\right|<\rho_{1}, \quad\left|1+\Gamma_{2}\right|<\rho_{2} \leqq\left|\Gamma_{2}\right|,
$$

are new, except for $\Gamma_{1}=\left(1+\Gamma_{2}\right)=0, \rho_{2}=1$, which is the theorem of Cowling, Leighton and Thron referred to earlier. The proof given for the best form of this result in [17] is so complicated that it seemed hopeless to obtain the extension to arbitrary $\Gamma_{1}, \Gamma_{2}$. Thus we believe that by making this important new result possible our method has proved its worth.

In 1956 Wall [20] gave a twin-convergence region type condition for the convergence of the even part of (1.1). Further results along this line were obtained by Perron [13] and one of the authors [17], [19]. An easy by-product of our method are numerous results of this type.

The methods used formerly in deriving twin-convergence regions $\left(E_{1}, E_{2}\right)$ have been quite varied. However, many of them make essential use of the assumption that there exist value regions $V_{1}$ and $V_{2}$ such that

$$
\frac{a_{2 n-1}}{1+V_{1}} \subseteq V_{2} \text { and } \quad \frac{a_{2 n}}{1+V_{2}} \subseteq V_{1} \text { if } a_{2 n-1} \in E_{1} \text { and } a_{2 n} \in E_{2}
$$

An elementary approach has been to observe that the sets $S_{n}\left(V_{k_{n}}\right)$, where $k_{n}=1$ if $n$ is odd and $k_{n}=2$ if $n$ is even, form a nested sequence of circular disks (for the definition of the functions $S_{n}$ see $\S 2$ ). This sequence can either shrink to a point, the limit point case, or to a disk, the limit circle case. If the limit point case holds for the continued fraction, then the continued fraction converges (at least if $0 \in V_{1} \cap V_{2}$ ). Thus in some instances [7], [9], [10], [14], [17] the strategy has been to show that the limit point case will occur under the conditions imposed on the elements of the continued fraction. This was done by estimating the radius $r_{n}$ of the $n$th nested disk. 
If the limit circle case occurs, the continued fraction may, or may not, converge. Our present strategy, previously used in [6], [8], [18], is to determine sufficient conditions for convergence of the continued fraction in the limit circle case, and to make sure that the element regions are so chosen as to incorporate these conditions. In this we make essential use of the basic fact that

$$
S_{n+1}(-1)=S_{n}(\infty)=S_{n-1}(0)
$$

holds for all continued fractions (1.1). It is for this reason that the location of the points $-1, \infty, 0$ with respect to the pre-value regions $V_{1}$ and $V_{2}$ plays such an important part in Theorem 4.3. It is noteworthy that the method employed in this article is both simple and elementary in the sense that no deep function-theoretic results are used. Moreover, by virtue of its wide range of applicability, the method provides a unified approach to the twin-convergence region problem.

Finally, a word on notation. By Int $(A), \bar{A}$ and $C(A)$ we mean the interior, closure and complement, respectively, of the set $A$ in the extended complex plane. If $f$ is a function of $k$ variables, we mean by $f\left(A_{1}, \ldots, A_{k}\right)$ the set

$$
\left\{f\left(x_{1}, \ldots, x_{k}\right): x_{m} \in A_{m}, m=1, \ldots, k\right\} .
$$

The symbol $\circ$ stands for functional composition; i.e. $f \circ g(z)=f[g(z)]$.

2. Basic concepts. In this section we summarize the definitions and basic properties of continued fractions that are needed. An (infinite) continued fraction is an ordered pair of sequences $\left[\left\{a_{n}\right\}_{n=1}^{\infty},\left\{f_{n}\right\}_{n=1}^{\infty}\right]$, where $a_{1}, a_{2}, \ldots$ are complex numbers, $a_{n} \neq 0, n=1,2, \ldots$, and where the $f_{n}$ are elements in the extended complex plane defined as follows: If $s_{n}$ denotes the linear fractional transformation (l.f.t.)

$$
s_{n}(z)=a_{n} /(1+z), \quad n=1,2, \ldots,
$$

and

$$
S_{1}(z)=s_{1}(z) ; \quad S_{n}(z)=S_{n-1}\left(s_{n}(z)\right), \quad n=2,3, \ldots
$$

then

$$
f_{n}=S_{n}(0), \quad n=1,2, \ldots
$$

The $a_{n}$ are called elements of the continued fraction $\left[\left\{a_{n}\right\},\left\{f_{n}\right\}\right]$ and $f_{n}$ is called the $n$th appproximant. A continued fraction is said to converge if its sequence of approximants converges and, in this case, $f=\lim f_{n}$ is called the value of the continued fraction. For convenience the continued fraction $\left[\left\{a_{n}\right\},\left\{f_{n}\right\}\right]$ is sometimes denoted by $(1.1), K_{n=1}^{\infty}\left(a_{n} / 1\right)$ or, more simply $K\left(a_{n} / 1\right)$, and the $n$th approximant by $K_{k=1}^{n}\left(a_{k} / 1\right)$. The symbols $(1.1), K_{n=1}^{\infty}\left(a_{n} / 1\right)$ and $K\left(a_{n} / 1\right)$ are also commonly used to denote the value of the continued fraction when it converges. The notion of a continued fraction as an ordered pair of sequences was suggested by Henrici and Pfluger [5]. 
The following standard formulas are stated for later reference:

$$
S_{n}(z)=\frac{A_{n-1} z+A_{n}}{B_{n-1} z+B_{n}}, \quad n=1,2, \ldots,
$$

where

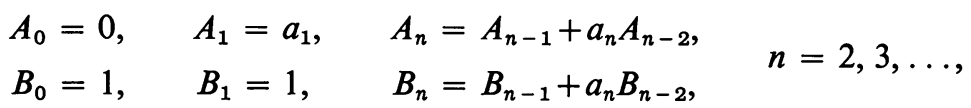

and

$$
A_{n} B_{n-1}-B_{n} A_{n-1}=(-1)^{n+1} \prod_{k=1}^{n} a_{k}
$$

Let $E_{1}$ and $E_{2}$ be sets of complex numbers. Nonempty sets $V_{1}$ and $V_{2}$ in the extended plane will be called pre-twin-value regions corresponding to the pair of sets $\left(E_{1}, E_{2}\right)$ if

$$
s\left(E_{1}, V_{1}\right) \subseteq V_{2}, \quad s\left(E_{2}, V_{2}\right) \subseteq V_{1},
$$

where

$$
s(a, z)=a /(1+z) .
$$

When this is the case, we shall write $\left(E_{1}, E_{2}\right)=m\left(V_{1}, V_{2}\right)$. We shall write $\left(E_{1}, E_{2}\right)$ $=M\left(V_{1}, V_{2}\right)$ if $\left(E_{1}, E_{2}\right)$ is a pair of maximal sets satisfying $(2.7)$; that is, if $\left(E_{1}^{*}, E_{2}^{*}\right)$ $=m\left(V_{1}, V_{2}\right)$ implies $E_{n}^{*} \subseteq E_{n}, n=1,2$. Pre-twin-value regions $V_{1}, V_{2}$ corresponding to $\left(E_{1}, E_{2}\right)$ are called twin-value regions corresponding to $\left(E_{1}, E_{2}\right)$ if

$$
E_{1} \subseteq V_{2}, \quad E_{2} \subseteq V_{1}
$$

Twin-value regions $V_{1}, V_{2}$ corresponding to $\left(E_{1}, E_{2}\right)$ are called best if $V_{1}, V_{2}$ are minimal; that is, if $V_{n} \subseteq V_{n}^{*}, n=1,2$, for every pair of twin value regions $V_{1}^{*}, V_{2}^{*}$ corresponding to $\left(E_{1}, E_{2}\right)$. It is easily seen that pre-twin-value regions $V_{1}, V_{2}$ corresponding to $\left(E_{1}, E_{2}\right)$ will be twin-value regions if $0 \in V_{1} \cap V_{2}$.

The characteristic property of twin-value regions $V_{1}, V_{2}$ corresponding to $\left(E_{1}, E_{2}\right)$ is that $V_{1}$ and $V_{2}$ contain all finite continued fractions

$$
\begin{aligned}
& \frac{a_{2}}{1}+\frac{a_{3}}{1}+\cdots+\frac{a_{m}}{1} ; \\
& \frac{a_{1}}{1}+\frac{a_{2}}{1}+\cdots+\frac{a_{m}}{1},
\end{aligned}
$$

respectively. Furthermore, $V_{1}$ and $V_{2}$ are best twin-value regions corresponding to $\left(E_{1}, E_{2}\right)$ if they contain no other elements. Thus the limit (if it exists) of a continued fraction $K\left(a_{n} / 1\right)$, with elements satisfying $a_{2 n-1} \in E_{1}, a_{2 n} \in E_{2}$, lies in $\bar{V}_{2}$, the closure of $V_{2}$. In this context the sets $\left(E_{1}, E_{2}\right)$ will be referred to as element regions (or more precisely, twin-element regions) for continued fractions of the 
form $K\left(a_{n} / 1\right)$. The properties stated above are immediate consequences of the following

THEOREM 2.1. Let $E_{1}$ and $E_{2}$ be sets of complex numbers. Let

$$
\begin{array}{r}
V_{n}=\left\{w: w=s_{n+1} \circ s_{n+2} \circ \cdots \circ s_{m}(0), m \geqq n+1, a_{2 k-1} \in E_{1}, a_{2 k} \in E_{2}\right\}, \\
n=0,1,
\end{array}
$$

where $s_{m}(z)=a_{m} /(1+z)$ and $V_{2}=V_{0}$. Then $V_{1}, V_{2}$ are best twin-value regions corresponding to $\left(E_{1}, E_{2}\right)$.

Proof. First we show that $V_{1}, V_{2}$ are twin-value regions corresponding to $\left(E_{1}, E_{2}\right)$. From (2.12) it follows that $a_{n+1}=s_{n+1}(0) \in V_{n}$ for all $a_{n+1} \in E_{n+1}, n=0,1$; hence (2.9) is satisfied. To verify (2.7) suppose that $w \in V_{n}$ and $a_{n} \in E_{n}, n=1$ or 2 . It follows from (2.12) that there exists an integer $m \geqq n+1$, and for each $k, n+1$ $\leqq k \leqq m$, there exist elements $a_{k} \in E_{k(\bmod 2)}$ such that

$$
w=s_{n+1} \circ s_{n+2} \circ \cdots \circ s_{m}(0) \text {. }
$$

Therefore (2.12) implies that $s_{n}(w) \in V_{n-1}$ and hence (2.7). Now to show that $V_{1}$ and $V_{2}$ are best, let $V_{1}^{*}, V_{2}^{*}$ denote arbitrary twin-value regions corresponding to $\left(E_{1}, E_{2}\right)$. It suffices to prove that $V_{n} \subseteq V_{n}^{*}, n=1,2$. It is easily shown by induction that for $n=1,2$ and $m \geqq n$

$$
s_{n} \circ s_{n+1} \circ \cdots \circ s_{m}\left(V_{m}^{*}(\bmod 2)\right) \subseteq V_{n-1}^{*},
$$

for arbitrary $a_{k} \in E_{k(\bmod 2)}$. Here we have set $V_{0}^{*}=V_{2}^{*}$. Since $E_{1} \subseteq V_{2}^{*}$ and $E_{2} \subseteq V_{1}^{*}$, $s_{m+1}(0)=a_{m+1} \in V_{m(\bmod 2)}^{*}$ for arbitrary $a_{m+1} \in E_{m+1(\bmod 2)}$. Combining this with (2.13) gives $s_{n} \circ \cdots \circ s_{m} \circ s_{m+1}(0) \in V_{n-1}^{*}$ and this completes the proof.

Throughout this paper we assume that the elements $a_{n}$ do not vanish. However, a few brief remarks can be made for the case when $a_{n}=0$ for some $n$. Suppose that $a_{m} \neq 0$ for $m<n$ but $a_{n}=0$. If $-1 \notin V_{1} \cup V_{2}$, then it follows that $S_{m}(0)=S_{n-1}(0)$ for all $m \geqq n$. Thus we obtain a "degenerate continued fraction" converging in a trivial manner. The condition $-1 \notin V_{1} \cup V_{2}$ is imposed in almost all of the convergence theorems to be given. The main exception to this is Theorem 4.3, Case 3(A) and results derived from it (part of Theorem 5.4 and Corollaries 5.7 and 5.8). If $-1 \in V_{1}$ but $-1 \notin V_{2}$, then $a_{2 n}=0$ can still be admitted in Case 3 of Theorem 4.3.

Finally, twin-convergence regions $\left(E_{1}, E_{2}\right)$ are said to be best if there do not exist twin-convergence regions $\left(E_{1}^{*}, E_{2}^{*}\right)$ such that

$$
E_{n} \subseteq E_{n}^{*}, \quad n=1,2,
$$

where containment is proper for at least one of the sets. It is well known that

$$
\sum_{n=1}^{\infty}\left|d_{n}\right|=\infty, \quad d_{1}=a_{1}^{-1}, \quad d_{n}=\left(d_{n-1} a_{n}\right)^{-1}, \quad n=2,3, \ldots,
$$

is a necessary condition for convergence of $K\left(a_{n} / 1\right)$. Thus $\left(E_{1}, E_{2}\right)$ are called conditional twin-convergence regions for continued fractions of the form $K\left(a_{n} / 1\right)$ 
if convergence is implied by (1.2) and (2.15) together. It follows from (2.15) that both members of a pair of twin-convergence regions cannot be unbounded. This is not true, however, of conditional twin-convergence regions. It also follows from (2.15) that subsets of conditional twin-convergence regions will be twin-convergence regions if at least one subset is bounded.

3. Necessary conditions. In the following sections we shall employ twin-value regions and, more generally, pre-twin value regions to obtain a large number of twin-convergence regions for continued fractions of the form $K\left(a_{n} / 1\right)$. It will be important to eliminate redundancies and convergence regions which are not best. For this purpose some helpful results are developed in the present section.

THEOREM 3.1. Let

$$
\left(E_{1}, E_{2}\right)=m\left(V_{1}, V_{2}\right) \text { and }\left(E_{1}^{*}, E_{2}^{*}\right)=m\left(W_{1}, W_{2}\right)
$$

where

$$
W_{1}=-1-C\left(V_{2}\right), \quad W_{2}=-1-C\left(V_{1}\right) .
$$

Then the following statements are true:

(A) $\left(E_{1}, E_{2}\right)=m\left(W_{1}, W_{2}\right)$ and $\left(E_{1}^{*}, E_{2}^{*}\right)=m\left(V_{1}, V_{2}\right)$,

(B) $\left(E_{1}, E_{2}\right)=M\left(V_{1}, V_{2}\right)$ and $\left(E_{1}^{*}, E_{2}^{*}\right)=M\left(W_{1}, W_{2}\right)$ imply $E_{n}=E_{n}^{*}, n=1,2$.

Proof. The transformation $v(z)=-1-z$ is idempotent (i.e. $v=v^{-1}$ ) and satisfies

$$
s_{n}=v \circ s_{n}^{-1} \circ v \text {, }
$$

where $s_{n}(z)=a_{n} /(1+z)$. Letting $V_{0}=V_{2}$ and $W_{0}=W_{2}$, we obtain for each $n=1,2$ and $a_{n} \in E_{n}$,

$$
s_{n}\left(W_{n}\right)=s_{n} \circ v\left[C\left(V_{n-1}\right)\right]=v \circ s_{n}^{-1}\left[C\left(V_{n-1}\right)\right] \subset v\left[C\left(V_{n}\right)\right]=W_{n-1} .
$$

Here we have used (3.2), (3.3) and the fact that (3.1) implies $s_{n}^{-1}\left[C\left(V_{n-1}\right)\right] \subseteq C\left(V_{n}\right)$. But (3.4) gives $\left(E_{1}, E_{2}\right)=m\left(W_{1}, W_{2}\right)$. The second part of (A) follows in a similar manner, since $V_{n}=-1-C\left(W_{n-1}\right), n=1,2$. (B) follows from (A) since $\left(E_{1}, E_{2}\right)$ and $\left(E_{1}^{*}, E_{2}^{*}\right)$ are maximal. This completes the proof.

REMARK. The preceding theorem shows that if $V_{1}, V_{2}$ are pre-twin value regions corresponding to twin element regions $\left(E_{1}, E_{2}\right)$, then $W_{1}, W_{2}$ defined by (3.2) are also pre-twin-value regions corresponding to $\left(E_{1}, E_{2}\right)$. This result is used extensively in the proof of Theorem 4.3.

THEOREM 3.2. If $V_{1}, V_{2}$ are best twin-value regions corresponding to nonempty element regions $\left(E_{1}, E_{2}\right)$, then at least one of the following must hold:

$$
-1 \in V_{1} \cup V_{2},
$$

or

$$
V_{1} \cap\left(-1-V_{2}\right)=\varnothing
$$


Proof. Let $W_{1}, W_{2}$ be defined by (3.2), so that $\left(E_{1}, E_{2}\right)=m\left(W_{1}, W_{2}\right)$. Suppose that

$$
E_{1} \subseteq W_{2} \text { and } E_{2} \subseteq W_{1},
$$

so that $W_{1}, W_{2}$ are twin-value regions corresponding to $\left(E_{1}, E_{2}\right)$. Since $V_{1}, V_{2}$ are best, we obtain $V_{2} \subseteq W_{2}=-1-C\left(V_{1}\right)$, which is equivalent to (3.6). On the other hand, suppose that (3.7) is false. Then for $n=1$ or 2 , there exists $a_{n} \in E_{n}$ such that $a_{n} \notin W_{n-1}=v\left[C\left(V_{n}\right)\right]$, where $v(z)=-1-z$ and $W_{0}=W_{2}$. It follows that $v\left(a_{n}\right) \in V_{n}$ and hence by Theorem $2.1 v\left(a_{n}\right)$ may be written in the form

$$
v\left(a_{n}\right)=s_{n+1} \circ \cdots \circ s_{m}(0), \quad a_{k} \in E_{k(\bmod 2)}, \quad m \geqq n+1 .
$$

But from (3.3) we obtain $v\left(a_{n}\right)=v \circ s_{n}(0)=s_{n}^{-1} \circ v(0)$ and hence

$$
v(0)=s_{n} \circ s_{n+1} \circ \cdots \circ s_{m}(0) \in V_{n-1}
$$

by Theorem 2.1. This implies (3.5) and completes the proof.

Theorem 3.2 gives a necessary condition for best twin-value regions corresponding to nonempty element regions. Our next theorem gives a necessary condition for best twin-value regions corresponding to conditional twin-convergence regions. The proof makes use of the following two lemmas. A proof of the first lemma may be found in [1, Theorem 2.1].

LEMMA 3.3. If the two periodic continued fractions

$$
\begin{aligned}
& \frac{a_{1}}{1}+\cdots+\frac{a_{n}}{1}+\frac{a_{1}}{1}+\cdots, \\
& \frac{a_{n}}{1}+\cdots+\frac{a_{1}}{1}+\frac{a_{n}}{1}+\cdots,
\end{aligned}
$$

converge to limits $w_{1}$ and $-\left(1+w_{2}\right)$, respectively, then $w_{1}$ and $w_{2}$ are the fixed points of the transformation $w=S_{n}(z)$ defined by (2.4).

LEMMA 3.4. If

$$
-1=\frac{a_{1}}{1}+\cdots+\frac{a_{m}}{1}, \quad a_{n} \neq 0, \quad 1 \leqq n \leqq m,
$$

then

$$
-1=\frac{a_{m}}{1}+\cdots+\frac{a_{1}}{1}
$$

Proof. Let $s_{n}(z)=a_{n} /(1+z), n=1, \ldots, m$, and $v(z)=-1-z$. Then from (3.10) and (3.3) we obtain

$$
v(0)=s_{1} \circ \cdots \circ s_{m}(0)=v \circ s_{1}^{-1} \circ \cdots \circ s_{m}^{-1} \circ v(0),
$$

from which (3.11) follows since $v=v^{-1}$. 
THEOREM 3.4. If $V_{1}, V_{2}$ are best twin-value regions corresponding to conditional twin-convergence regions $\left(E_{1}, E_{2}\right)$, then

$$
-1 \notin V_{1} \cap V_{2} .
$$

Proof. Assuming $-1 \in V_{1} \cap V_{2}$ we shall prove the existence of at least one divergent periodic continued fraction $K\left(a_{n} / 1\right)$ with elements satisfying $a_{n} \in E_{n(\bmod 2)}$, $a_{n} \neq 0$. This is sufficient for our purpose since a periodic continued fraction always satisfies (2.15). It follows from the assumption and from Theorem 2.1 that there exist integers $n$ and $k$ and there exist nonzero elements $a_{1}, \ldots, a_{n}$ and $d_{2}, \ldots, d_{k}$ such that

$$
\begin{array}{ll}
-1=\frac{a_{1}}{1}+\cdots+\frac{a_{n}}{1}, & a_{m} \in E_{m(\bmod 2)} \\
-1=\frac{d_{2}}{1}+\cdots+\frac{d_{k}}{1}, & d_{m} \in E_{m(\bmod 2)} .
\end{array}
$$

It will suffice to consider the following three cases: (1) If $n$ is even, we consider the periodic continued fractions of period $n+2$

$$
\frac{a_{1}}{1}+\cdots+\frac{a_{n}}{1}+\frac{x_{1}}{1}+\frac{x_{2}}{1}+\frac{a_{1}}{1}+\cdots
$$

and

$$
\frac{x_{2}}{1}+\frac{x_{1}}{1}+\frac{a_{n}}{1}+\cdots+\frac{a_{1}}{1}+\frac{x_{2}}{1}+\cdots,
$$

where $x_{1}$ and $x_{2}$ denote arbitrary nonzero elements of $E_{1}$ and $E_{2}$, respectively. (2) If $k$ is $o d d$, we consider instead the periodic continued fractions of period $k+1$

$$
\frac{d_{2}}{1}+\cdots+\frac{d_{k}}{1}+\frac{z_{2}}{1}+\frac{z_{1}}{1}+\cdots+\frac{d_{2}}{1}+\cdots
$$

and

$$
\frac{z_{1}}{1}+\frac{z_{2}}{1}+\frac{d_{k}}{1}+\cdots+\frac{d_{2}}{1}+\frac{z_{1}}{1}+\cdots
$$

where $z_{1}$ and $z_{2}$ denote arbitrary nonzero elements of $E_{1}$ and $E_{2}$, respectively. (3) If $n$ is odd and $k$ is even, and if $y_{1} \in E_{1}$ and $y_{2} \in E_{2}\left(y_{i} \neq 0\right)$, then

$$
-1=\frac{a_{1}}{1}+\cdots+\frac{a_{n}}{1}+\frac{y_{2}}{1}+\frac{y_{1}}{1}+\frac{d_{2}}{1}+\cdots+\frac{d_{k}}{1}
$$

involves an even number of elements and hence could be used to construct periodic continued fractions of the same type considered in case (1). We shall show that in case (1) either (3.14) or (3.15) diverges for some $x_{1} \in E_{1}, x_{2} \in E_{2}\left(x_{i} \neq 0\right)$. The proof for case (2) is completely analogous.

The continued fractions (3.14) and (3.15) are so constructed that the approximants of order $n+m(n+2)$ of the first are all -1 and the approximants of order 
$m(n+2)$ of the second are all zero. The last statement follows from Lemma 3.4. Since $E_{1}, E_{2}$ are conditional twin-convergence regions, the continued fractions must converge to -1 and 0 , respectively. Thus by Lemma 3.3, $w_{1}=-1$ and $w_{2}$ $=-1$ are the fixed points of the transformation $w=S_{n+2}(z)$ defined by (2.4) and (2.5). It follows that the quadratic equation

$$
B_{n+1} w^{2}+\left(B_{n+2}-A_{n+1}\right) w-A_{n+2}=0
$$

has a double root -1 , and so we must have

$$
A_{n+2} / B_{n+1}=-1 \text { and }\left(B_{n+2}-A_{n+1}\right) / B_{n+1}=2 \text {. }
$$

But from (2.5) we have $A_{n+2}=A_{n+1}+x_{2} A_{n}$ and $B_{n+2}=B_{n+1}+x_{2} B_{n}$. Hence (3.19) becomes

$$
x_{2} B_{n}=\left(A_{n+1}+B_{n+1}\right) \text { and } x_{2} A_{n}=-\left(A_{n+1}+B_{n+1}\right) .
$$

These equations can hold for more than one $x_{2}$ if and only if $A_{n}=B_{n}=0$. But from (2.6)

$$
A_{n+1} B_{n}-A_{n} B_{n+1}=a_{1} a_{2} \cdots a_{n} x_{1} \neq 0 .
$$

Hence there is only one choice for $x_{2}$ and so $E_{2}$ must consist of only one point. From (3.20) it follows that $x_{2}\left(A_{n}+B_{n}\right)=0$ and so $A_{n}+B_{n}=0$ and $A_{n} B_{n} \neq 0$. Again using (3.20) and (2.5) we obtain

$$
x_{2}=\frac{A_{n+1}+B_{n+1}}{B_{n}}=\frac{\left(A_{n}+x_{1} A_{n-1}\right)+\left(B_{n}+x_{1} B_{n-1}\right)}{B_{n}}=x_{1} \frac{A_{n-1}+B_{n-1}}{B_{n}} .
$$

It follows that there is but one choice for $x_{1}$ and so $E_{1}$ must consist of only one point. But if $E_{1}$ and $E_{2}$ both consist of only one point, then the continued fraction (3.14) is of period 2. Thus its approximants of order $(m+1)(n+2)$ are all 0 and so the continued fraction diverges. This completes the proof.

Our use of value regions and pre-value regions in the remaining part of this paper will be restricted to regions of the following three types: (1) circular disks, (2) complements of disks, and (3) half-planes. The point at infinity is always in the interior of the complement of a disk and on the boundary of a half-plane. There are a number of other such conditions which must be satisfied by pre-twin-value regions (of the types listed above) corresponding to nonempty element regions $\left(E_{1}, E_{2}\right)$. These are summarized in Table 1 for later use in $\S 4$.

Remarks. The results listed in Table 1 may be easily verified by considering the image of $z=\infty$ under the transformation $s(a, z)$. It is easily seen that cases (4) and (5) may be reduced to cases (2) and (1), respectively, by using the corresponding pre-twin-value regions $W_{1}, W_{2}$ defined by (3.2).

4. General convergence criteria. We shall now establish a method by which a large number of twin-convergence regions may be derived. Our main convergence result is given in Theorem 4.3; however, in a remark following that theorem it is 


\begin{tabular}{|c|l|l|l|l|}
\hline & \multicolumn{2}{|c|}{ Pre-twin-value regions } & \multicolumn{2}{c|}{ Necessary conditions for } \\
\hline Case & \multicolumn{1}{|c|}{$V_{1}$} & \multicolumn{1}{c|}{$V_{2}$} & $s\left(E_{1}, V_{1}\right) \subseteq V_{2}$ & \multicolumn{1}{c|}{$s\left(E_{2}, V_{2}\right) \subseteq V_{1}$} \\
\hline 1 & disk & disk & $-1 \notin \bar{V}_{1}$ & $-1 \notin \bar{V}_{2}$ \\
\hline 2 & disk & half-plane & $-1 \notin$ Int $V_{1}$ & $0 \in \bar{V}_{1},-1 \notin \bar{V}_{2}$ \\
\hline 3 & disk & $\begin{array}{c}\text { complement } \\
\text { of disk }\end{array}$ & & $0 \in$ Int $V_{1},-1 \notin \bar{V}_{2}$ \\
\hline 4 & half-plane & $\begin{array}{c}\text { complement } \\
\text { of disk }\end{array}$ & $0 \in \bar{V}_{2}$ & $0 \in$ Int $V_{1},-1 \notin$ Int $V_{2}$ \\
\hline 5 & $\begin{array}{c}\text { complement } \\
\text { of disk }\end{array}$ & $\begin{array}{c}\text { complement } \\
\text { of disk }\end{array}$ & $0 \in$ Int $V_{2}$ & $0 \in$ Int $V_{1}$ \\
\hline 6 & half-plane & half-plane & $0 \in \bar{V}_{2},-1 \notin$ Int $V_{1}$ & $0 \in \bar{V}_{1},-1 \notin$ Int $V_{2}$ \\
\hline
\end{tabular}

TABLE 1. Necessary conditions for pre-twin-value regions $V_{1}, V_{2}$ corresponding to nonempty element regions $\left(E_{1}, E_{2}\right)$. Note: $s(a, z)=a /(1+z)$.

pointed out that other convergence regions are obtainable from the method. The proof of the main theorem is based on the following two lemmas which deal with convergence of sequences of linear fractional transformations.

LEMMA 4.1. Let $\left\{T_{n}\right\}$ be a sequence of l.f.t.'s of the form

$$
T_{n}(z)=C_{n}+R_{n}\left(z+\bar{G}_{n}\right) /\left(G_{n} z+1\right), \quad n=1,2, \ldots,
$$

satisfying

$$
\left|R_{n}\right|=r_{n} \searrow r>0, \quad\left|C_{n}-C_{n-1}\right| \leqq r_{n-1}-r_{n}, \quad\left|G_{n}\right|=g_{n}<1 .
$$

(A) Suppose that there exists a sequence of points $\left\{\zeta_{n}\right\}$ in the extended complex plane such that

$$
T_{2 n+1}\left(\zeta_{2 n+1}\right)=T_{2 n}\left(\zeta_{2 n}\right), \quad\left|\zeta_{2 n+1}\right| \geqq 1, \quad\left|\zeta_{2 n}\right| \leqq 1, \quad n \geqq 1 .
$$

If for some $\varepsilon>0,\left|\zeta_{2 n+1}\right| \geqq 1+\varepsilon, n \geqq 1$, then

$$
\sum_{n=1}^{\infty}\left(1-g_{2 n+1}\right)<\infty .
$$

If for some $\varepsilon>0,\left|\zeta_{2 n}\right| \leqq 1-\varepsilon, n \geqq 1$, then

$$
\sum_{n=1}^{\infty}\left(1-g_{2 n}\right)<\infty .
$$

(B) Suppose that there exists a sequence of points $\left\{\zeta_{n}^{\prime}\right\}$ in the extended complex plane such that

$$
T_{2 n}\left(\zeta_{2 n}^{\prime}\right)=T_{2 n-1}\left(\zeta_{2 n-1}^{\prime}\right), \quad\left|\zeta_{2 n}^{\prime}\right| \geqq 1, \quad\left|\zeta_{2 n-1}^{\prime}\right| \leqq 1, \quad n \geqq 1 .
$$


If for some $\varepsilon>0,\left|\zeta_{2 n}^{\prime}\right| \geqq 1+\varepsilon, n \geqq 1$, then (4.5) holds. If for some $\varepsilon>0,\left|\zeta_{2 n-1}^{\prime}\right| \leqq 1-\varepsilon$, $n \geqq 1$, then (4.4) holds.

Proof. Consider case (A) in which (4.3) is satisfied. From (4.1) and (4.3) we obtain

$$
C_{2 n+1}+R_{2 n+1} \lambda_{2 n+1}=C_{2 n}+R_{2 n} \lambda_{2 n}
$$

where

$$
\lambda_{n}=\left(\zeta_{n}+\bar{G}_{n}\right) /\left(G_{n} \zeta_{n}+1\right) .
$$

We note that $\left\{\lambda_{2 n+1}\right\}$ is bounded. This follows from (4.7) and the fact that $r_{n} \searrow r>0$, $\left\{C_{n}\right\}$ converges and $\left|\lambda_{2 n}\right| \leqq 1$. From (4.2) and (4.7) we obtain

$$
r_{2 n+1}\left|\lambda_{2 n+1}\right| \leqq r_{2 n}-r_{2 n+1}+r_{2 n}\left|\lambda_{2 n}\right|
$$

and hence

$$
r_{2 n+1} / r_{2 n} \leqq 1-H_{n},
$$

where $0 \leqq H_{n}=\left(\left|\lambda_{2 n+1}\right|-\left|\lambda_{2 n}\right|\right) /\left(1+\left|\lambda_{2 n+1}\right|\right)<1$. It follows from (4.8) and $r_{2 n} / r_{2 n-1}$ $\leqq 1$ that

$$
r_{2 n+1} \leqq L \prod_{k=1}^{n}\left(1-H_{k}\right)
$$

where $L$ is a constant independent of $n$. Therefore the series $\sum H_{n}$ converges, for otherwise the infinite product $\Pi\left(1-H_{n}\right)$ would diverge to zero and thus contradict the hypothesis $r_{n} \searrow r>0$. Since $\left\{\lambda_{2 n+1}\right\}$ is bounded, we conclude that $\sum\left(\left|\lambda_{2 n+1}\right|-\left|\lambda_{2 n}\right|\right)$ converges and hence both of the series

$$
\sum\left(\left|\lambda_{2 n+1}\right|-1\right) \text { and } \sum\left(1-\left|\lambda_{2 n}\right|\right)
$$

converge, since $\left|\lambda_{2 n+1}\right| \geqq 1$ and $\left|\lambda_{2 n}\right| \leqq 1$.

Now suppose that for some $\varepsilon>0,\left|\zeta_{2 n}\right| \leqq 1-\varepsilon, n \geqq 1$. In this case it suffices to show that

$$
\left(1-g_{2 n}\right) K \leqq 1-\left|\lambda_{2 n}\right|, \quad n \geqq 1,
$$

for some positive constant $K$ sufficiently small. But for $0<K<\frac{1}{2},(4.11)$ is equivalent to

$$
\left|\zeta_{2 n}+\bar{G}_{2 n}\right| \leqq\left[1-K\left(1-g_{2 n}\right)\right]\left|G_{2 n} \zeta_{2 n}+1\right| .
$$

Squaring both sides of (4.12), collecting terms and dividing by $\left(1-g_{2 n}\right)$, we obtain the equivalent inequality

$$
K\left[2-K\left(1-g_{2 n}\right)\right]\left|G_{2 n} \zeta_{2 n}+1\right|^{2} \leqq\left(1-\left|\zeta_{2 n}\right|^{2}\right)\left(1+g_{2 n}\right) .
$$

The right side of this inequality is positive and uniformly bounded from zero. The left side is bounded above by $8 K$. Hence if $K>0$ is sufficiently small, (4.13) will hold for all $n$. It follows that (4.11) and hence also (4.5) are satisfied. By a 
similar argument it can be shown that (4.4) holds if $\left|\zeta_{2 n+1}\right| \geqq 1+\varepsilon, n \geqq 1$. Case (B) follows from (A) by a change of index. This completes the proof.

LEMMA 4.2. Let $\left\{T_{n}\right\}$ be a sequence of l.f.t.'s of the form (4.1), (4.2).

(A) Suppose that there exist sequences of points $\left\{\xi_{n}\right\}$ and $\left\{\eta_{n}\right\}$ in the extended complex plane such that for some $\varepsilon>0$

$$
T_{2 n+1}\left(\xi_{n}\right)=T_{2 n-1}\left(\eta_{n}\right), \quad|| \xi_{n}|-1| \geqq \varepsilon, \quad|| \eta_{n}|-1| \geqq \varepsilon, \quad n \geqq 1 .
$$

If $\sum\left(1-g_{2 n-1}\right)<\infty$, then $\left\{T_{2 n+1}(z)\right\}$ converges for all $z$ in the extended plane such that $|z| \neq 1$ and

$$
\lim T_{2 n+1}(z)=\lim \left[C_{2 n+1}+R_{2 n+1} / G_{2 n+1}\right], \quad|z| \neq 1 .
$$

(B) Suppose that there exist sequences of points $\left\{\xi_{n}^{\prime}\right\}$ and $\left\{\eta_{n}^{\prime}\right\}$ in the extended complex plane such that for some $\varepsilon>0$

$$
T_{2 n+2}\left(\xi_{n}^{\prime}\right)=T_{2 n}\left(\eta_{n}^{\prime}\right), \quad|| \xi_{n}^{\prime}|-1| \geqq \varepsilon, \quad|| \eta_{n}^{\prime}|-1| \geqq \varepsilon, \quad n \geqq 1 .
$$

If $\sum\left(1-g_{2 n}\right)<\infty$, then $\left\{T_{2 n}(z)\right\}$ converges for all $z$ in the extended plane such that $|z| \neq 1$ and

$$
\lim T_{2 n}(z)=\lim \left[C_{2 n}+R_{2 n} / G_{2 n}\right], \quad|z| \neq 1 .
$$

Proof. Case (A). From (4.14) we obtain

$$
\begin{aligned}
R_{2 n+1} \bar{G}_{2 n+1}-R_{2 n-1} \bar{G}_{2 n-1}= & \left(C_{2 n-1}-C_{2 n+1}\right)-R_{2 n+1} \xi_{n} \frac{1-g_{2 n+1}^{2}}{G_{2 n+1} \xi_{n}+1} \\
& +R_{2 n-1} \eta_{n} \frac{1-g_{2 n-1}^{2}}{G_{2 n-1} \eta_{n}+1} .
\end{aligned}
$$

From summing equations of this form we obtain for $m<n$

$$
\begin{aligned}
R_{2 n+1} \bar{G}_{2 n+1}-R_{2 m+1} \bar{G}_{2 m+1}= & \left(C_{2 m+1}-C_{2 n+1}\right)-\sum_{k=m+1}^{n} R_{2 k+1} \frac{1-g_{2 k+1}^{2}}{G_{2 k+1}+1 / \xi_{k}} \\
& +\sum_{k=m+1}^{n} R_{2 k-1} \frac{1-g_{2 k-1}^{2}}{G_{2 k-1}+1 / \eta_{k}} .
\end{aligned}
$$

Thus it is readily seen that $\left\{R_{2 n+1} \bar{G}_{2 n+1}\right\}$ will be a Cauchy sequence provided $\sum\left(1-g_{2 n+1}\right)<\infty$. For then we have $g_{2 n+1} \rightarrow 1$ so that by (4.14) the sequences

$$
\left\{G_{2 k+1}+1 / \xi_{k}\right\}, \quad\left\{G_{2 k-1}+1 / \eta_{k}\right\}
$$

are bounded from zero for large enough $k$. The proof of (A) is completed by observing that (4.1) may be written in the form

$$
T_{n}(z)=C_{n}+\frac{R_{n}}{G_{n}}\left[1-\frac{1-g_{n}^{2}}{G_{n} z+1}\right]
$$

It suffices to note that (B) follows from (A) by a change of index. We mention here that it would be possible to weaken the hypothesis of Lemma 4.2 to allow 
$\left|\xi_{n}\right| \rightarrow 1$ and $\left|\eta_{n}\right| \rightarrow 1$. However, the resulting conditions would be far more complicated, and such a strengthening of the lemma has not been needed. We are now ready to state and prove the main convergence theorem.

THEOREM 4.3. Let $V_{1}, V_{2}$ be pre-twin-value regions corresponding to a pair of element regions $\left(E_{1}, E_{2}\right)$, where $V_{1}$ and $V_{2}$ are regions in the extended plane of the following types: circular disks, complements of disks or half-planes. $V_{1}$ and $V_{2}$ may contain all, none or part of their boundaries. Let $K\left(a_{n} / 1\right)$ be a continued fraction with elements satisfying

$$
a_{2 n-1} \in E_{1}, \quad a_{2 n} \in E_{2}, \quad a_{n} \neq 0, \quad n=1,2, \ldots,
$$

and let $f_{n}$ denote its $n$th approximant. Then the following statements are true:

Case 1. Let $V_{1}$ and $V_{2}$ be disks. Then $K\left(a_{n} / 1\right)$ converges if $0 \notin \partial V_{1}, 0 \in \bar{V}_{2}$ and $-1 \notin \bar{V}_{1} \cup \bar{V}_{2}$.

Case 2. Let $V_{1}$ be a disk and $V_{2}$ be a half-plane. Then

(A) $K\left(a_{n} / 1\right)$ converges if $0 \in$ Int $V_{1}$ and $-1 \notin \bar{V}_{1} \cup \bar{V}_{2}$.

(B) $\left\{f_{2 n-1}\right\}$ converges if one of the following holds:

$$
0 \in \bar{V}_{1} \cap \text { Int } V_{2} \text { and }-1 \notin \text { Int } V_{1} \cup \bar{V}_{2} \text {. }
$$

Case 3. Let $V_{1}$ be a disk and $V_{2}$ be the complement of a disk. Then

(A) $K\left(a_{n} / 1\right)$ converges if $0 \in$ Int $V_{1} \cap \bar{V}_{2}$ and $-1 \notin \partial V_{1} \cup \bar{V}_{2}$,

(B) $\left\{f_{2 n-1}\right\}$ converges if $0 \in$ Int $V_{1}$ and $-1 \notin \bar{V}_{1} \cup \bar{V}_{2}$.

Proof. Let $v_{1}$ and $v_{2}$ be linear fractional transformations with the properties

$$
v_{k}\left(\bar{V}_{k}\right)=U, \quad k=1,2,
$$

where $U$ denotes the closed disk $|z| \leqq 1$. Let $v_{2 n+1}=v_{1}, v_{2 n}=v_{2}, n=0,1, \ldots$, and let $V_{0}=V_{2}$. Define sequences of 1.f.t.'s $\left\{t_{n}\right\}$ and $\left\{T_{n}\right\}$ by

$$
t_{n}=v_{n-1} \circ s_{n} \circ v_{n}^{-1}, \quad n=1,2, \ldots,
$$

and

$$
T_{1}=t_{1} ; \quad T_{n}=T_{n-1} \circ t_{n}, \quad n=2,3, \ldots,
$$

where $s_{n}(z)=s\left(a_{n}, z\right)=a_{n} /(1+z)$. It follows from (2.7) and (4.22) that

$$
T_{n}(U) \subseteq T_{n-1}(U) \subseteq U, \quad n=2,3, \ldots
$$

Thus as shown in [18], $T_{n}(z)$ has the form (4.1) satisfying (4.2) with the additional possibility of $r=0$. It is further shown that $C=\lim C_{n}$ exists and that $\left\{T_{n}(U)\right\}$ is a nested sequence of closed disks; $C_{n}$ is the center and $r_{n}$ the radius of $T_{n}(U)$. The case with $r_{n} \searrow r=0$ is referred to as the limit point case, since $\left\{T_{n}(U)\right\}$ converges to the point $C$. When $r_{n} \searrow r>0,\left\{T_{n}(U)\right\}$ converges to the closed disk with radius $r$ and center $C$. This is called the limit circle case. 
From (2.2) and (4.22) we obtain

$$
f_{n}=S_{n}(0)=v_{0}^{-1} \circ T_{n}\left[v_{n}(0)\right],
$$

so that $\left\{f_{n}\right\}$ converges if and only if $\left\{T_{n}\left[v_{n}(0)\right]\right\}$ converges. Thus in particular $\left\{f_{n}\right\}$ will converge if the limit point case occurs and if $0 \in \bar{V}_{1} \cap \bar{V}_{2}$ since then $\left|v_{n}(0)\right| \leqq 1$, $n=1,2$. It also follows from (2.1) and (2.2) that

$$
S_{n}(-1)=S_{n-1}(\infty)=S_{n-2}(0), \quad n=3,4, \ldots,
$$

from which we have

$$
\begin{aligned}
& T_{2 n+1}\left[v_{1}(-1)\right]=T_{2 n}\left[v_{2}(\infty)\right]=T_{2 n-1}\left[v_{1}(0)\right], \\
& T_{2 n+2}\left[v_{2}(-1)\right]=T_{2 n+1}\left[v_{1}(\infty)\right]=T_{2 n}\left[v_{2}(0)\right],
\end{aligned} \quad n=1,2, \ldots
$$

Now consider Case 1, with $V_{1}$ and $V_{2}$ both disks and suppose (a) $0 \in \operatorname{Int} V_{1}$. In view of the preceding remarks it suffices to consider the limit circle case. To this end let $\zeta_{2 n-1}^{\prime}=\xi_{n}=v_{1}(-1), \zeta_{2 n}^{\prime}=v_{2}(\infty)$ and $\eta_{n}=v_{1}(0), n=1,2, \ldots$ Since $\left|v_{1}(-1)\right|$ $>1,\left|v_{2}(\infty)\right|>1$ and $\left|v_{1}(0)\right|<1$, it follows from Lemma 4.1 (B) that $\sum\left(1-g_{2 n-1}\right)<\infty$ and hence from Lemma 4.2(A) that $\left\{T_{2 n+1}(z)\right\}$ converges for all $z$ such that $|z| \neq 1$. Thus from (4.15) and (4.27)

$$
\begin{aligned}
\lim T_{2 n-1}\left[v_{1}(0)\right] & =\lim \left[C_{2 n+1}+R_{2 n+1} / G_{2 n+1}\right] \\
& =\lim T_{2 n+1}\left[v_{1}(\infty)\right]=\lim T_{2 n}\left[v_{2}(0)\right] ;
\end{aligned}
$$

whence $\left\{f_{n}\right\}$ converges. In the alternative situation (b) $0 \notin \bar{V}_{1}$, we consider the corresponding pair of pre-twin-value regions $W_{1}, W_{2}$ defined by (3.2). Here $W_{1}$ and $W_{2}$ are both complements of disks and it is easily verified that

$$
0 \in \text { Int } W_{1} \cap \text { Int } W_{2}, \quad-1 \notin \text { Int } W_{1}, \quad-1 \in \text { Int } W_{2} \text {. }
$$

Now we choose l.f.t.'s $v_{1}$ and $v_{2}$ with the properties

$$
v_{k}\left(\bar{W}_{k}\right)=U, \quad k=1,2,
$$

and proceed as above. Again it suffices to consider the limit circle case and we let $\zeta_{2 n+1}=v_{1}(-1), \zeta_{2 n}=v_{2}(\infty), \xi_{n}^{\prime}=v_{2}(-1)$ and $\eta_{n}^{\prime}=v_{2}(0), n=1,2, \ldots$ Since $\left|v_{1}(-1)\right|$ $\geqq 1,\left|v_{2}(\infty)\right|<1,\left|v_{2}(-1)\right|<1$ and $\left|v_{2}(0)\right|<1$ it follows from Lemma 4.1(A) that $\sum\left(1-g_{2 n}\right)<\infty$ so that from Lemma 4.2(B) $\left\{T_{2 n}(z)\right\}$ converges for $z$ such that $|z| \neq 1$ and (4.17) holds. Thus using (4.26) we have

$$
\begin{aligned}
\lim T_{2 n}\left[v_{2}(0)\right] & =\lim \left[C_{2 n}+R_{2 n} / G_{2 n}\right] \\
& =\lim T_{2 n}\left[v_{2}(\infty)\right]=\lim T_{2 n-1}\left[v_{1}(0)\right],
\end{aligned}
$$

so that $\left\{f_{n}\right\}$ converges.

Next we consider Case 2 with $V_{1}$ a disk and $V_{2}$ a half-plane. First suppose that

$$
0 \in \operatorname{Int} V_{1} \cap \bar{V}_{2} \text { and }-1 \notin \bar{V}_{1} \cup \bar{V}_{2}
$$


Letting $v_{1}$ and $v_{2}$ be 1.f.t.'s satisfying (4.20), we proceed as in Case 1. Again it suffices to consider the limit circle case and we set $\zeta_{2 n+1}=\xi_{n}=v_{1}(-1), \zeta_{2 n}=v_{2}(\infty)$ and $\eta_{n}=v_{1}(0), n=1,2, \ldots$ Since $\left|v_{1}(-1)\right|>1,\left|v_{2}(\infty)\right|=1$ and $\left|v_{1}(0)\right|<1$, it follows from Lemma 4.1(A) that $\sum\left(1-g_{2 n+1}\right)<\infty$ and hence from Lemma 4.2(A) that $\left\{T_{2 n+1}(t)\right\}$ converges for $|z| \neq 1$ and that (4.15) holds. Therefore using (4.27) and $\left|v_{1}(\infty)\right|>1$, we obtain

$$
\begin{aligned}
\lim T_{2 n+1}\left[v_{1}(0)\right] & =\lim \left[C_{2 n+1}+R_{2 n+1} / G_{2 n+1}\right] \\
& =\lim T_{2 n+1}\left[v_{1}(\infty)\right]=\lim T_{2 n}\left[v_{2}(0)\right] ;
\end{aligned}
$$

whence $\left\{f_{n}\right\}$ converges. The alternative situation under Case $2(\mathrm{~A})$ is that

$$
0 \in \text { Int } V_{1}, \quad 0 \notin \bar{V}_{2}, \quad-1 \notin \bar{V}_{1} \cup \bar{V}_{2} \text {. }
$$

In this situation we use the alternate pre-twin-value regions $W_{1}, W_{2}$ defined by (3.2), which satisfy

$$
0 \in \text { Int } W_{1} \cap \text { Int } W_{2}, \quad-1 \in \text { Int } W_{1} \quad \text { and } \quad-1 \notin \bar{W}_{2} \text {. }
$$

Here $W_{1}$ is a half-plane and $W_{2}$ the complement of a disk. As in Case 1 we choose 1.f.t.'s $v_{1}$ and $v_{2}$ with the properties (4.28) and it suffices to consider the limit circle case. Let $\zeta_{2 n+1}=v_{1}(\infty), \zeta_{2 n}=\eta_{n}^{\prime}=v_{2}(0)$ and $\xi_{n}^{\prime}=v_{2}(-1), n=1,2, \ldots$ Since $\left|v_{1}(\infty)\right|$ $=1,\left|v_{2}(0)\right|<1$ and $\left|v_{2}(-1)\right|<1$, it follows from Lemma 4.1(A) that $\sum\left(1-g_{2 n}\right)<\infty$ and hence from Lemma $4.2(\mathrm{~B})$ that $\left\{T_{2 n}(z)\right\}$ converges for $|z| \neq 1$ and that (4.17) holds. Therefore from (4.26) and $\left|v_{2}(\infty)\right|<1$ we obtain

$$
\begin{aligned}
\lim T_{2 n}\left[v_{2}(0)\right] & =\lim \left[C_{2 n}+R_{2 n} / G_{2 n}\right] \\
& =\lim T_{2 n}\left[v_{2}(\infty)\right]=\lim T_{2 n-1}\left[v_{1}(0)\right],
\end{aligned}
$$

so that $\left\{f_{n}\right\}$ converges. This proves Case 2(A). In the proof of Case 2(B) we proceed as above using the pre-twin-value regions $W_{1}$ and $W_{2}$. The properties of $W_{1}, W_{2}$ corresponding to Case 2(B) are

$$
0 \in \text { Int } W_{1} \cap \bar{W}_{2} \text { and }-1 \notin \bar{W}_{1} \cup \text { Int } W_{2} \text {. }
$$

In this situation it suffices to consider the limit circle case and we let $\zeta_{2 n+1}=\xi_{n}$ $=v_{1}(-1), \zeta_{2 n}=v_{2}(\infty)$ and $\eta_{n}=v_{1}(0), n=1,2, \ldots$. Since $\left|v_{1}(-1)\right|>1,\left|v_{2}(\infty)\right|<1$ and $\left|v_{1}(0)\right|<1$, it follows from Lemma 4.1(A) that $\sum\left(1-g_{2 n+1}\right)<\infty$ so that from Lemma 4.2(A) $\left\{T_{2 n+1}(z)\right\}$ converges for $|z| \neq 1$. Therefore

$$
\lim T_{2 n+1}\left[v_{1}(0)\right]=\lim \left[C_{2 n+1}+R_{2 n+1} / G_{2 n+1}\right],
$$

and we deduce the convergence of $\left\{f_{2 n+1}\right\}$. In this situation we are not able to conclude that $\left\{T_{2 n}\left[v_{2}(0)\right]\right\}$ converges as in the previous cases considered, since now $\left|v_{1}(\infty)\right|=1$.

Finally, we come to Case 3, where $V_{1}$ is a disk and $V_{2}$ the complement of a disk. Again we let $v_{1}$ and $v_{2}$ denote 1.f.t.'s satisfying (4.20) and proceed as in the previous cases. In part (A) it suffices to consider the limit circle case. Let $\zeta_{2 n+1}=v_{1}(\infty)$, 
$\zeta_{2 n}=v_{2}(0), \xi_{n}=v_{1}(-1)$ and $\eta_{n}=v_{1}(0), n=1,2, \ldots$ Then since $\left|v_{1}(\infty)\right|>1,\left|v_{2}(0)\right|$ $\leqq 1,\left|v_{1}(-1)\right| \neq 1$ and $\left|v_{1}(0)\right|<1$, it follows from Lemma 4.1(A) that $\sum\left(1-g_{2 n-1}\right)$ $<\infty$ and hence by Lemma 4.2(A) $\left\{T_{2 n+1}(z)\right\}$ converges for $|z| \neq 1$ and (4.15) holds. Thus, using (4.27) and $\left|v_{1}(\infty)\right|>1$, we obtain

$$
\begin{aligned}
\lim T_{2 n+1}\left[v_{1}(0)\right] & =\lim \left[C_{2 n+1}+R_{2 n+1} / G_{2 n+1}\right] \\
& =\lim T_{2 n+1}\left[v_{1}(\infty)\right]=\lim T_{2 n}\left[v_{2}(0)\right] ;
\end{aligned}
$$

whence $\left\{f_{n}\right\}$ converges. In the proof of part (B) we are able to deduce only the convergence of $\left\{f_{2 n-1}\right\}$ in the limit point case, since no assumptions are made regarding $v_{2}(0)$. The limit circle case is handled by the same methods used above. Let $\zeta_{2 n+1}$ $=\xi_{n}=v_{1}(-1), \zeta_{2 n}=v_{2}(\infty)$ and $\eta_{n}=v_{1}(0), n=1,2, \ldots$ Then since $\left|v_{1}(-1)\right|>1$, $\left|v_{2}(\infty)\right|<1$ and $\left|v_{1}(0)\right|<1$, we obtain from Lemma 4.1(A) that $\sum\left(1-g_{2 n-1}\right)<\infty$ and hence from Lemma 4.2(A) that $\left\{T_{2 n+1}(z)\right\}$ converges for $|z| \neq 1$. Therefore $\left\{T_{2 n+1}\left[v_{1}(0)\right]\right\}$ converges and so also $\left\{f_{2 n-1}\right\}$. This completes the proof of Theorem 4.3.

REMARKS CONCERNING THEOREM 4.3. In stating conditions sufficient for convergence we have eliminated those cases which fail to satisfy the necessary conditions for nonempty element regions listed in Table 1 . We have tried to give an exhaustive set of twin-convergence criteria for continued fractions of the form $K\left(a_{n} / 1\right)$ obtainable from Lemmas 4.1 and 4.2 applied to (4.25). Some additional twinconvergence criteria could be obtained by applying the method to the equations

$$
S_{n+1}\left(-1-a_{n+1}\right)=S_{n}(-1)=S_{n-1}(\infty)=S_{n-2}(0)=S_{n-3}\left(a_{n-2}\right),
$$

and introducing additional restrictions on the elements $a_{n}$. It is also possible to obtain convergence criteria from Lemmas 4.1 and 4.2 applied to a sequence of value regions $\left\{V_{n}\right\}$. A number of results of this type have already been given in [7], [8]. Without further modification the method does not give twin-convergence criteria for the case with $V_{1}$ and $V_{2}$ both half-planes. This is due mainly to the fact that infinity is on the boundaries of these regions so that we get $\left|v_{n}(\infty)\right|=1, n=1,2$, in the notation of (4.20). However, this case has been extensively developed in the two preceding references. The cases with $V_{1}$ a half-plane, $V_{2}$ the complement of a disk and with $V_{1}, V_{2}$ both complements of disks are included in Cases 2 and 1, respectively. This is done by using the alternate pre-twin-value regions $W_{1}, W_{2}$ defined by (3.2). This allows a more concise formulation of the results and the elimination of certain ambiguities.

5. Twin-convergence regions. This section is devoted to the development of explicit twin-convergence regions obtained from Theorem 4.3.

THEOREM 5.1 (DISK-DISK CASE). A continued fraction $K\left(a_{n} / 1\right)$ converges if its elements satisfy

$$
a_{2 n-1} \in E_{1}, \quad a_{2 n} \in E_{2}, \quad a_{n} \neq 0, \quad n=1,2,3, \ldots,
$$


where

$$
\begin{array}{rlrl}
E_{n}=\left\{w:\left|w\left(1+\bar{\Gamma}_{n}\right)-\Gamma_{n-1}\left(\left|1+\Gamma_{n}\right|^{2}-\rho_{n}^{2}\right)\right|+\rho_{n}|w|\right. & \\
& \left.\leqq \rho_{n-1}\left(\left|1+\Gamma_{n}\right|^{2}-\rho_{n}^{2}\right)\right\}, & n=1,2, \\
\left|\Gamma_{1}\right| \neq \rho_{1}<\left|1+\Gamma_{1}\right|, \quad\left|\Gamma_{2}\right| \leqq \rho_{2}<\left|1+\Gamma_{2}\right|, \quad \Gamma_{0}=\Gamma_{2}, & \rho_{0}=\rho_{2} .
\end{array}
$$

Proof. It follows from Lemma 2.1 of [8] that $V_{1}, V_{2}$ defined by

$$
V_{n}=\left\{z:\left|z-\Gamma_{n}\right| \leqq \rho_{n}\right\}, \quad n=1,2 \quad\left(V_{0}=V_{2}\right)
$$

are pre-twin-value regions corresponding to the element regions $\left(E_{1}, E_{2}\right)$. Thus the theorem is a direct result of Theorem 4.3, Case 1 .

TheOREM 5.2 (DISK-HALF-PLANE CASE). A continued fraction $K\left(a_{n} / 1\right)$ converges if its elements satisfy (5.1), where

$$
\begin{gathered}
E_{1}=\left\{w:|w|[\rho-|1+\Gamma| \cos (\arg w-\arg (1+\Gamma)-\psi)] \leqq p\left[|1+\Gamma|^{2}-\rho^{2}\right]\right\} \\
E_{2}=\left\{w:\left|w-2 \Gamma e^{i \psi}(\cos \psi-p)\right|+|w| \leqq 2 \rho(\cos \psi-\rho)\right\} \\
|\Gamma|<\rho<|1+\Gamma|, \quad 0 \leqq p<\cos \psi
\end{gathered}
$$

The sequence of odd approximants $\left\{f_{2 n-1}\right\}$ converges if 'the elements satisfy (5.1), (5.5) and (5.6) with

$$
|\Gamma| \leqq \rho \leqq|1+\Gamma|, \quad 0<p<\cos \psi .
$$

Remarks. The element regions $E_{1}$ and $E_{2}$ are bounded by a hyperbola and an ellipse, respectively. The proof of the theorem is an immediate consequence of Theorem 4.3, Case 2 and the following lemma.

LEMMA 5.3. Let $V_{1}$ be a closed disk and $V_{2}$ a closed half-plane defined by

$$
\begin{aligned}
& V_{1}=\{z:|z-\Gamma| \leqq \rho\}, \quad|\Gamma| \leqq \rho \leqq|1+\Gamma|, \\
& V_{2}=\left\{z: \operatorname{Re}\left(z e^{-i \psi}\right) \geqq-p\right\}, \quad 0 \leqq p<\cos \psi .
\end{aligned}
$$

Then $V_{1}, V_{2}$ are pre-twin-value regions corresponding to the element regions $\left(E_{1}, E_{2}\right)$ defined by (5.5), (5.6).

Proof. It suffices to verify (2.7). First we note that

$$
s\left(w, V_{2}\right)=\left\{z:\left|z-D_{2}\right| \leqq q_{2}\right\}
$$

where

$$
D_{2}=w e^{-i \psi} / 2(\cos \psi-p), \quad q_{2}=|w| / 2(\cos \psi-p) .
$$

Thus $s\left(w, V_{2}\right) \subseteq V_{1}$ if and only if $\left|D_{2}-\Gamma\right|+q_{2} \leqq \rho$, which is equivalent to the inequality in (5.6) and which further proves that $E_{2}$ is maximal. Next we consider two separate cases.

(1) Suppose $\rho<|1+\Gamma|$. Then $s\left(w, V_{1}\right)=\left\{z:\left|z-D_{1}\right| \leqq q_{1}\right\}$, where

$$
D_{1}=w(1+\bar{\Gamma}) /\left(|1+\Gamma|^{2}-\rho^{2}\right), \quad q_{1}=\rho|w| /\left(|1+\Gamma|^{2}-\rho^{2}\right) .
$$


Thus a necessary and sufficient condition for $s\left(w, V_{1}\right) \subseteq V_{2}$ is that

$$
\operatorname{Re}\left[D_{1} e^{-i \psi}\right]+p \geqq q_{1},
$$

which is equivalent to the inequality in (5.5) and therefore proves that $E_{1}$ is maximal.

(2) In the alternate case, $\rho=|1+\Gamma|, s\left(w, V_{1}\right)$ becomes the half-plane

$$
s\left(w, V_{1}\right)=\{z: \operatorname{Re}[z \exp [-i(\arg w-\arg (1+\Gamma))]] \geqq|w| /(2|1+\Gamma|)\} .
$$

Therefore we will have $s\left(w, V_{1}\right) \subseteq V_{2}$ if and only if

$$
\arg w=\arg (1+\Gamma)+\psi .
$$

But the inequality in (5.5) reduces to (5.11) when $\rho=|1+\Gamma|$. This completes the proof.

THEOREM 5.4 (DISK-COMPLEMENT OF DISK CASE). A continued fraction $K\left(a_{n} / 1\right)$ converges if its elements satisfy (5.1), where either

$$
\begin{aligned}
& E_{1}=\left\{w:\left|w\left(1+\bar{\Gamma}_{1}\right)+\Gamma_{2}\left(\rho_{1}^{2}-\left|1+\Gamma_{1}\right|^{2}\right)\right|-\rho_{1}|w| \geqq \rho_{2}\left(\left|1+\Gamma_{1}\right|^{2}-\rho_{1}^{2}\right)\right\}, \\
& E_{2}=\left\{w:\left|w\left(1+\bar{\Gamma}_{2}\right)+\Gamma_{1}\left(\rho_{2}^{2}-\left|1+\Gamma_{2}\right|^{2}\right)\right|+\rho_{2}|w| \leqq \rho_{1}\left(\rho_{2}^{2}-\left|1+\Gamma_{2}\right|^{2}\right)\right\}
\end{aligned}
$$$$
\text { or } E_{2} \text { is defined by (5.13) but }
$$

$$
E_{1}=\left\{w: \rho_{1}|w|-\left|w\left(1+\bar{\Gamma}_{1}\right)+\Gamma_{2}\left(\rho_{1}^{2}-\left|1+\Gamma_{1}\right|^{2}\right)\right| \geqq \rho_{2}\left(\rho_{1}^{2}-\left|1+\Gamma_{1}\right|^{2}\right)\right\}
$$

and

$$
\left|\Gamma_{1}\right|<\rho_{1}, \quad\left|1+\Gamma_{1}\right|<\rho_{1}, \quad\left|1+\Gamma_{2}\right|<\rho_{2} \leqq\left|\Gamma_{2}\right| .
$$

The sequence of odd approximates $\left\{f_{2 n-1}\right\}$ converges if the elements satisfy (5.1), (5.12) and (5.13), where

$$
\left|\Gamma_{1}\right|<\rho_{1}<\left|1+\Gamma_{1}\right|, \quad\left|1+\Gamma_{2}\right|<\rho_{2} .
$$

REMARKs. Again the proof is an immediate consequence of Theorem 4.3, Case 3 and the following lemma:

LEMMA 5.5. Let $V_{1}$ and $V_{2}$ be defined by

$$
\begin{aligned}
& V_{1}=\left\{z:\left|z-\Gamma_{1}\right| \leqq \rho_{1}\right\}, \quad\left|\Gamma_{1}\right|<\rho_{1} \neq\left|1+\Gamma_{1}\right|, \\
& V_{2}=\left\{z:\left|z-\Gamma_{2}\right| \geqq \rho_{2}\right\}, \quad\left|1+\Gamma_{2}\right|<\rho_{2} .
\end{aligned}
$$

If $\rho_{1}<\left|1+\Gamma_{1}\right|$, then $V_{1}, V_{2}$ are pre-twin-value regions corresponding to $\left(E_{1}, E_{2}\right)$ defined by (5.12) and (5.13). If $\left|1+\Gamma_{1}\right|<\rho_{1}$, then $V_{1}, V_{2}$ are pre-twin-value regions corresponding to $\left(E_{1}, E_{2}\right)$ defined by (5.15) and (5.13).

Proof. It is easily shown that $s\left(w, V_{2}\right)$ is the circular disk $\left|z-D_{2}\right| \leqq q_{2}$, where $D_{2}=-w\left(1+\bar{\Gamma}_{2}\right) /\left[\rho_{2}^{2}-\left|1+\Gamma_{2}\right|^{2}\right]$ and $q_{2}=\rho_{2}|w| /\left[\rho_{2}^{2}-\left|1+\Gamma_{2}\right|^{2}\right]$. Thus $s\left(w, V_{2}\right) \subseteq V_{1}$ if and only if $\left|D_{2}-\Gamma_{1}\right|+q_{2} \leqq \rho_{1}$, which is equivalent to the inequality in (5.13) 
and therefore shows that $E_{2}$ is maximal. If $\rho_{1}<\left|1+\Gamma_{1}\right|$, then $s\left(w, V_{1}\right)$ is the disk $\left|z-D_{1}\right| \leqq q_{1}$, where $D_{1}=w\left(1+\bar{\Gamma}_{1}\right) /\left[\left|1+\Gamma_{1}\right|^{2}-\rho_{1}^{2}\right]$ and $q_{1}=\rho_{1}|w| /\left[\left|1+\Gamma_{1}\right|^{2}-\rho_{1}^{2}\right]$. Thus $s\left(w, V_{1}\right) \subseteq V_{2}$ if and only if $\left|D_{1}-\Gamma_{2}\right| \geqq q_{1}+\rho_{2}$, which is equivalent to the inequality in (5.12) and thus proves that $E_{1}$ is maximal. On the other hand, if $\left|1+\Gamma_{1}\right|<\rho_{1}$, then $s\left(w, V_{1}\right)=\left\{z:\left|z-D_{1}^{\prime}\right| \geqq q_{1}^{\prime}\right\}$, where $D_{1}^{\prime}=w\left(1+\bar{\Gamma}_{1}\right) /\left[\left|1+\Gamma_{1}\right|^{2}-\rho_{1}^{2}\right]$ and $q_{1}^{\prime}=\rho_{1}|w| /\left[\rho_{1}^{2}-\left|1+\Gamma_{1}\right|^{2}\right]$. Thus $s\left(w, V_{1}\right) \subseteq V_{2}$ if and only if $\left|D_{1}^{\prime}-\Gamma_{2}\right|+\rho_{2} \leqq q_{1}^{\prime}$, which is equivalent to the inequality in (5.15) and which further proves that $E_{1}$ is maximal. This completes the proof.

The following corollary is an interesting special case of Theorem 5.4, obtained by using (5.12) and (5.13) with $\rho_{1}=\rho_{2}=\rho$ and $\Gamma_{1}=-\left(1+\Gamma_{2}\right)=\Gamma$. It was proved in 1960 by Lange and Thron [16]. We note in passing that for the case of real $\Gamma$, $\Gamma>-\frac{1}{2}$, they were able to prove that the convergence is uniform over the element regions and to obtain useful estimates of the speed of convergence. Lange [9] has subsequently been able to extend uniform convergence to include complex values of $\Gamma$ with $\operatorname{Re}(\Gamma)>-\frac{1}{2}$.

COROLlary 5.6. A continued fraction $K\left(a_{n} / 1\right)$ converges if its elements satisfy (5.1), where

$$
\begin{gathered}
E_{1}=\left\{w:\left|w(1+\bar{\Gamma})-(1+\Gamma)\left(\rho^{2}-|1+\Gamma|^{2}\right)\right|-\rho|w| \geqq \rho\left[|1+\Gamma|^{2}-\rho^{2}\right]\right\} \\
E_{2}=\left\{w:\left|w \bar{\Gamma}-\Gamma\left(\rho^{2}-|\Gamma|^{2}\right)\right|+\rho|w| \leqq \rho\left[\rho^{2}-|\Gamma|^{2}\right]\right\} \\
|\Gamma|<\rho<|1+\Gamma|
\end{gathered}
$$

Remarks. The notation used here differs slightly from that of Lange and Thron. A brief explanation will aid in comparing the results. We must verify that the twinconvergence regions (1.3) are the same as (5.20) and (5.21). For this it suffices to consider the corresponding value regions. The element regions (5.20) and (5.21) are determined by

$$
s\left(E_{1}, V_{1}\right) \subseteq V_{2}, \quad s\left(E_{2}, V_{2}\right) \subseteq V_{1}, \quad \text { where } s(a, z)=a /(1+z),
$$

and where the value regions $V_{1}, V_{2}$ are given by

$$
V_{1}=\{z:|z-\Gamma| \leqq \rho\}, \quad V_{2}=\{z:|z+1+\Gamma| \geqq \rho\}, \quad|\Gamma|<\rho<|1+\Gamma| .
$$

The element regions $\left(E_{1}^{*}, E_{2}^{*}\right)$ of $(1.3)$ are determined by

$$
s^{*}\left(E_{1}^{*}, V_{1}^{*}\right) \subseteq V_{2}^{*}, \quad s^{*}\left(E_{2}^{*}, V_{2}^{*}\right) \subseteq V_{1}^{*}, \quad \text { where } s^{*}(a, z)=1+a / z,
$$

and where the value regions $V_{1}^{*}, V_{2}^{*}$ are given by $V_{n}^{*}=1+V_{n}, n=1,2$. Now it is easily verified that $s^{*}(a, z)=f^{-1} \circ s(a, f(z))$, where $f(z)=z-1$. It follows that $s^{*}\left(a, V_{1}^{*}\right) \subseteq V_{2}^{*}$ if and only if $s\left(a, V_{1}\right) \subseteq V_{2}$, so that $E_{1}^{*}=E_{1}$. Similarly we obtain $E_{2}^{*}=E_{2}$.

Another important special case of Theorem 5.4 is obtained by setting $\Gamma_{1}=$ $-\left(1+\Gamma_{2}\right)=\Gamma, \rho_{1}=\rho$ and $\rho_{2}=|1+\Gamma|$ in (5.13) and (5.15). We state this as 
Corollary 5.7. A continued fraction $K\left(a_{n} / 1\right)$ converges if its elements satisfy (5.1), where

$$
\begin{gathered}
E_{1}=\left\{w: \rho|w|-\left|w(1+\bar{\Gamma})-(1+\Gamma)\left(\rho^{2}-|1+\Gamma|^{2}\right)\right| \geqq|1+\Gamma|\left[\rho^{2}-|1+\Gamma|^{2}\right]\right\}, \\
E_{2}=\left\{w:|1+\Gamma||w|+\left|w \bar{\Gamma}-\Gamma\left(|1+\Gamma|^{2}-|\Gamma|^{2}\right)\right| \leqq \rho\left[|1+\Gamma|^{2}-|\Gamma|^{2}\right]\right\}, \\
|\Gamma|<|1+\Gamma|<\rho .
\end{gathered}
$$

Remarks. When $\Gamma=0$, Corollary 5.7 reduces to the following result of Thron [17]:

COROLlaRY 5.8. A continued fraction $K\left(a_{n} / 1\right)$ converges if for all $n=1,2,3, \ldots$ the elements $a_{n}$ satisfy $a_{n} \neq 0$ and

$$
\begin{gathered}
\left|a_{2 n-1}\right| \geqq 2\left[\rho-\cos \left(\arg a_{2 n-1}\right)\right], \\
\left|a_{2 n}\right| \leqq \rho,
\end{gathered}
$$

where $\rho>1$.

6. Elimination of twin-convergence regions which are not best. Let $C$ be a given family of twin-convergence regions for continued fractions of the form $K\left(a_{n} / 1\right)$. A subfamily $C^{*}$ of $C$ will be said to have property $P$ relative to the family $C$ if for each pair $\left(E_{1}, E_{2}\right) \in C$, there exists a pair $\left(E_{1}^{*}, E_{2}^{*}\right) \in C^{*}$ such that

$$
E_{i} \subseteq E_{1}^{*}, \quad i=1,2 .
$$

Property $\mathrm{P}$ does not insure that $C^{*}$ will contain best twin-convergence regions; it does imply that a pair of twin-convergence regions $\left(E_{1}, E_{2}\right)$ cannot be best if $\left(E_{1}, E_{2}\right)$ belong to $C$ but not $C^{*}$. In this section we investigate the problem: Given a family $C$ of twin-convergence regions, can we determine a proper subfamily $C^{*}$ with property $\mathrm{P}$ ? This is a difficult problem for which we have so far been able to obtain only some initial results. Our approach has been to select a subfamily $C^{*}$ by means of an intelligent guess and then determine whether $C^{*}$ has property $\mathrm{P}$ relative to $C$.

In the remainder of this paper we shall refer to $C$ as the family of all twinconvergence regions $\left(E_{1}, E_{2}\right)$ of the form $(5.12),(5.13)$, where the parameters $\Gamma_{i}, \rho_{i}$ satisfy

$$
\left|\Gamma_{1}\right|<\rho_{1}<\left|1+\Gamma_{1}\right|, \quad\left|1+\Gamma_{2}\right|<\rho_{2}<\left|\Gamma_{2}\right|
$$

and

$$
\left|\Gamma_{1}+1+\Gamma_{2}\right|+\rho_{1} \leqq \rho_{2} .
$$

Twin-value regions $V_{1}, V_{2}$ corresponding to such $\left(E_{1}, E_{2}\right)$ are given by the open regions

$$
V_{1}=\left\{z:\left|z-\Gamma_{1}\right|<\rho_{1}\right\}, \quad V_{2}=\left\{z:\left|z-\Gamma_{2}\right|>\rho_{2}\right\}
$$


To see this, note that by Lemma $5.5 \bar{V}_{1}, \bar{V}_{2}$ are pre-twin-value regions corresponding to $\left(E_{1}, E_{2}\right)$. But $s\left(E_{1}, \bar{V}_{1}\right) \subseteq \bar{V}_{2}$ and $s\left(E_{2}, \bar{V}_{2}\right) \subseteq \bar{V}_{1}$ imply $s\left(E_{1}\right.$, Int $\left.V_{1}\right) \subseteq V_{2}$ and $s\left(E_{2}\right.$, Int $\left.V_{2}\right) \subseteq$ Int $V_{1}$. Thus $V_{1}, V_{2}$ are twin-value regions, since $0 \in V_{1} \cap V_{2}$. We note further that since $-1 \notin \bar{V}_{1} \cup \bar{V}_{2}$, it follows from Theorem 3.2 that $V_{1}$, $V_{2}$ cannot be best twin-value regions corresponding to $\left(E_{1}, E_{2}\right)$ unless $V_{1} \cap$ $-\left(1+V_{2}\right)=\varnothing$; that is, unless (6.3) holds.

By $C^{*}$ we shall denote the subfamily of $C$ consisting of all twin-convergence regions $\left(E_{1}^{*}, E_{2}^{*}\right)$ obtained from $(5.12)$ and (5.13) by replacing $E_{i}, \Gamma_{i}$ and $\rho_{i}$ by $E_{i}^{*}, \Gamma_{i}^{*}$ and $\rho_{i}^{*}$, respectively, where

$$
\Gamma=\Gamma_{1}^{*}=-\left(1+\Gamma_{2}^{*}\right), \quad \rho=\rho_{1}^{*}=\rho_{2}^{*}, \quad|\Gamma|<\rho<|1+\Gamma| .
$$

The subfamily $C^{*}$ consists of the twin-convergence regions obtained by Corollary 5.6. We have made the conjecture that $C^{*}$ has property $\mathrm{P}$ relative to $C$; however, we are able to verify this conjecture only in two special situations given by Theorems 6.2 and 6.6. The question in general remains open.

We begin by describing the regions $\left(E_{1}, E_{2}\right)$ defined by (5.12) and (5.13), in terms of polar coordinates. Let $w=r e^{i \theta}, r \geqq 0$. Then $w \in E_{1}$ if and only if

$$
r^{2}-2\left[\rho_{1} \rho_{2}-\left|\Gamma_{2}\left(1+\Gamma_{1}\right)\right| \cos \left(\theta-\varphi_{1}\right)\right] r+\left(\left|1+\Gamma_{1}\right|^{2}-\rho_{1}^{2}\right)\left(\left|\Gamma_{2}\right|^{2}-\rho_{2}^{2}\right) \geqq 0
$$

and if $w \in E_{2}$ then

$$
r^{2}-2\left[\rho_{1} \rho_{2}-\left|\Gamma_{1}\left(1+\Gamma_{2}\right)\right| \cos \left(\theta-\varphi_{2}\right)\right] r+\left(\rho_{1}^{2}-\left|\Gamma_{1}\right|^{2}\right)\left(\rho_{2}^{2}-\left|1+\Gamma_{2}\right|^{2}\right) \geqq 0,
$$

where

$$
\varphi_{1}=\arg \left[-\Gamma_{2}\left(1+\Gamma_{1}\right)\right], \quad \varphi_{2}=\arg \left[-\Gamma_{1}\left(1+\Gamma_{2}\right)\right] .
$$

We note that $\arg w=\varphi_{i}$ is an axis of symmetry of $E_{i}, i=1,2$, and that $0 \in \operatorname{Int} E_{1}$ $\cap$ Int $E_{2} . E_{1}$ is unbounded and $E_{2}$ is bounded; both are connected sets. In deriving (6.6) from (5.13) we introduced an extraneous unbounded region which will subsequently be removed from the description of $E_{2}$. If $\Gamma_{1}\left(1+\Gamma_{2}\right) r \neq 0$, then (6.5) and (6.6) may be written in the form

$$
\cos \left(\theta-\varphi_{i}\right) \geqq h_{i}(r) \equiv a_{i} / r+b_{i}+c_{i} r, \quad i=1,2,
$$

where

$$
\begin{array}{ll}
a_{1}=\frac{-\left(\left|1+\Gamma_{1}\right|^{2}-\rho_{1}^{2}\right)\left(\left|\Gamma_{2}\right|^{2}-\rho_{2}^{2}\right),}{2\left|\Gamma_{2}\left(1+\Gamma_{1}\right)\right|} & a_{2}=\frac{-\left(\rho_{1}^{2}-\left|\Gamma_{1}\right|^{2}\right)\left(\rho_{2}^{2}-\left|1+\Gamma_{2}\right|^{2}\right)}{2\left|\Gamma_{1}\left(1+\Gamma_{2}\right)\right|}, \\
b_{1}=\rho_{1} \rho_{2} /\left|\Gamma_{2}\left(1+\Gamma_{1}\right)\right|, & b_{2}=\rho_{1} \rho_{2} /\left|\Gamma_{1}\left(1+\Gamma_{2}\right)\right|, \\
c_{1}=-1 / 2\left|\Gamma_{2}\left(1+\Gamma_{1}\right)\right|, & c_{2}=-1 / 2\left|\Gamma_{1}\left(1+\Gamma_{2}\right)\right| .
\end{array}
$$

Since the $a_{i}$ and $c_{i}$ are negative, $y=h_{i}(r)$ defines a hyperbola which tends to $-\infty$ as $r \rightarrow 0_{+}$and as $r \rightarrow+\infty$; the asymptotes are $r=0$ and $y=b_{i}+c_{i} r$. Letting

$$
y_{i}(\max )=\max _{r>0} h_{i}(r), \quad i=1,2,
$$


we obtain

$$
\begin{aligned}
-1<y_{1}(\max ) & =\frac{\rho_{1} \rho_{2}-\left[\left(\left|1+\Gamma_{1}\right|^{2}-\rho_{1}^{2}\right)\left(\left|\Gamma_{2}\right|^{2}-\rho_{2}^{2}\right)\right]^{1 / 2}}{\left|\Gamma_{2}\left(1+\Gamma_{1}\right)\right|}<1, \\
y_{2}(\max ) & =\frac{\rho_{1} \rho_{2}-\left[\left(\rho_{1}^{2}-\left|\Gamma_{1}\right|^{2}\right)\left(\rho_{2}^{2}-\left|1+\Gamma_{2}\right|^{2}\right)\right]^{1 / 2}}{\left|\Gamma_{1}\left(1+\Gamma_{2}\right)\right|} \geqq 1 .
\end{aligned}
$$

Thus $y=h_{i}(r)$ crosses the line $y=-1$ at two distinct points which we denote by $r_{i}^{-}$and $r_{i}^{+}$with $0<r_{i}^{-}<r_{i}^{+}$and

$$
r_{1}^{ \pm}=\left(\rho_{1} \pm\left|1+\Gamma_{1}\right|\right)\left(\rho_{2} \pm\left|\Gamma_{2}\right|\right), \quad r_{2}^{ \pm}=\left(\rho_{1} \pm\left|\Gamma_{1}\right|\right)\left(\rho_{2} \pm\left|1+\Gamma_{2}\right|\right) .
$$

Now let $H_{1}$ and $H_{2}$ be the regions defined by

$$
\begin{aligned}
& H_{1}=\left\{(r, y):|y| \leqq 1 \text { and either } r=0 \text { or } r>0 \text { and } y \geqq h_{1}(r)\right\}, \\
& H_{2}=\left\{(r, y):|y| \leqq 1 \text { and either } r=0 \text { or } 0<r \leqq r_{2}(\max ) \text { and } y \geqq h_{2}(r)\right\},
\end{aligned}
$$

where $r_{2}(\max )$ is defined by $h_{2}\left(r_{2}(\max )\right)=y_{2}(\max )$ and where $(r, y)$ are rectangular coordinates. Then

$$
w=r e^{i \theta} \in E_{i}, \quad i=1,2,
$$

if and only if $\left(r, \cos \left(\theta-\varphi_{i}\right)\right) \in H_{i}, i=1,2$. Except for the points on the axis of symmetry of $E_{i}$, there exists a two-to-one correspondence between the points of $E_{i}$ and the points of $H_{i}$. The extraneous unbounded region introduced in (6.6) corresponds to the unbounded region determined by $|y| \leqq 1, r>r_{2}(\max ), y \geqq h_{2}(r)$.

In the following we shall use the symbols $\varphi_{i}^{*}, a_{i}^{*}, b_{i}^{*}, c_{i}^{*}, h_{i}^{*}(r), y_{i}^{*}(\max ), r_{i}^{*}(\max )$, $r_{i}^{* \pm}$ and $H_{i}^{*}$ to denote the quantities obtained from the corresponding expressions without the asterisk $\left(^{*}\right)$ by replacing the parameters $\Gamma_{i}, \rho_{i}$ by $\Gamma_{i}^{*}, \rho_{i}^{*}$. We have thus obtained the following

TheOREM 6.1. Let $\left(E_{1}, E_{2}\right) \in C$ and $\left(E_{1}^{*}, E_{2}^{*}\right) \in C^{*}$. Then

$$
E_{i} \subseteq E_{i}^{*}, \quad i=1,2,
$$

provided

$$
\varphi_{i}=\varphi_{i}^{*} \text { and } H_{i} \subseteq H_{i}^{*}, \quad i=1,2 .
$$

REMARKS. There are two cases in which the condition $\varphi_{i}=\varphi_{i}^{*}, i=1,2$, is easily obtained. One is when $\Gamma_{i}=\Gamma_{i}^{*}, i=1,2$ (the concentric case) and the other is when $\Gamma_{1},-\left(1+\Gamma_{2}\right)$ and $\Gamma_{1}^{*}=-\left(1+\Gamma_{2}^{*}\right)$ are all positive so that $\varphi_{i}=\varphi_{i}^{*}=0, i=1,2$. Thus in these two special situations, for which we have obtained some positive results, it will suffice for our purpose to verify the second condition in (6.15). Our first result is

THEOREM 6.2. Let $\left(E_{1}, E_{2}\right) \in C$ where the parameters $\Gamma_{i}, \rho_{i}$ satisfy the additional conditions

$$
\begin{gathered}
0<\Gamma_{1}<\rho_{1}<1+\Gamma_{1}, \quad 0<-\left(1+\Gamma_{2}\right)<\rho_{2}<-\Gamma_{2}, \\
\left(\rho_{1}-\Gamma_{1}\right)^{1 / 2} / \Gamma_{1} \leqq\left(\rho_{2}+1+\Gamma_{2}\right)^{1 / 2} /-\left(1+\Gamma_{2}\right) .
\end{gathered}
$$


Let $\left(E_{1}^{*}, E_{2}^{*}\right) \in C^{*}$, where the parameters $\Gamma_{i}^{*}, \rho_{i}^{*}$ are given by

$$
\begin{array}{lll}
\Gamma=\Gamma_{1}^{*}=-\left(1+\Gamma_{2}^{*}\right)=A /(B-1), & \rho=\rho_{1}^{*}=\rho_{2}^{*}=B \Gamma, \\
A=\left[\left(\rho_{1}-\Gamma_{1}\right)\left(\rho_{2}+1+\Gamma_{2}\right)\right]^{1 / 2}, & B=\frac{1}{2}\left[\rho_{1} / \Gamma_{1}-\rho_{2} /\left(1+\Gamma_{2}\right)\right] .
\end{array}
$$

Then

$$
0<\Gamma<\rho<1+\Gamma
$$

and

$$
E_{i} \subseteq E_{i}^{*}, \quad i=1,2 .
$$

Remarks. The condition $0<A<1$ follows from the hypothesis $0<\rho_{1}-\Gamma_{1}<1$, $0<\rho_{2}+1+\Gamma_{2}<1$. To see that $B>1$ note that

$$
B-1=\frac{\Gamma_{1}\left(\rho_{2}+1+\Gamma_{2}\right)-\left(1+\Gamma_{2}\right)\left(\rho_{1}-\Gamma_{1}\right)}{-2 \Gamma_{1}\left(1+\Gamma_{2}\right)}>0 \text {. }
$$

Thus we obtain $0<\Gamma<\rho$ from (6.18) and $\rho=B \Gamma<1+\Gamma$ from $\Gamma=A /(B-1)$ $<1 /(B-1)$. Our proof of Theorem 6.2 will be based on the following lemmas.

LEMMA 6.3. The following statements follow from the hypothesis of Theorem 6.2:

(a) $r_{2}^{-}=r_{2}^{*-}$ and the curves $y=h_{2}(r)$ and $y=h_{2}^{*}(r)$ are tangent to each other at this point.

(b) $r_{2}^{*+} \leqq r_{2}^{+}$.

(c) $h_{2}^{*}(r) \leqq h_{2}(r)$ for $r_{2}^{*-} \leqq r \leqq r_{2}^{*+}$ and hence $E_{2} \subseteq E_{2}^{*}$.

(d) $r_{1}^{-} \leqq r_{1}^{*-} \leqq r_{1}^{*+} \leqq r_{1}^{+}$.

Proof. It is easily verified that $r_{2}^{-}=r_{2}^{*-}$ if and only if

$$
\rho-\Gamma=\left[\left(\rho_{1}-\Gamma_{1}\right)\left(\rho_{2}+1+\Gamma_{2}\right)\right]^{1 / 2}=A .
$$

Then by an elementary calculation we may show that $h_{2}^{\prime}\left(r_{2}^{-}\right)=h_{2}^{* \prime}\left(r_{2}^{*-}\right)$ if and only if

$$
\rho / \Gamma=\frac{1}{2}\left[\rho_{1} / \Gamma_{1}-\rho_{2} /\left(1+\Gamma_{2}\right)\right]=B .
$$

Combining (6.21) and (6.22) gives (6.18) and hence proves (a). Part (b) follows from the fact that $1=y_{2}^{*}(\max ) \leqq y_{2}(\max ) \geqq 1$ and that the two hyperbolas $y=h_{2}(r)$, $y=h_{2}^{*}(r)$ intersect in at most two points, both of which in this case occur at $r=r_{2}^{-}$ $=r_{2}^{*-}$. Part (c) follows from (a), (b) and Theorem 6.1, since $\varphi_{2}=\varphi_{2}^{*}=0$ and $H_{2} \subseteq H_{2}^{*}$. To prove (d) first note that

$$
r_{1}^{-}=\left[\rho_{1}-\left(1+\Gamma_{1}\right)\right]\left(\rho_{2}+\Gamma_{2}\right) \leqq(\rho-(1+\Gamma)]^{2}=r_{1}^{*-}
$$

if and only if

$$
\left[\left[\rho_{1}-\left(1+\Gamma_{1}\right)\right]\left(\rho_{2}+\Gamma_{2}\right)\right]^{1 / 2} \leqq 1+\Gamma-\rho=1-\left[\left(\rho_{1}-\Gamma_{1}\right)\left(\rho_{2}+1+\Gamma_{2}\right)\right]^{1 / 2} .
$$

Here the last equality follows from (6.21). It is easily shown that (6.24) is equivalent to $\left[\left(\rho_{1}-\Gamma_{1}\right)^{1 / 2}-\left(\rho_{2}+1+\Gamma_{2}\right)^{1 / 2}\right]^{2} \geqq 0$, so that (6.23) is true. Now since $r_{2}^{*+} \leqq r_{2}^{+}$ implies that $\rho+\Gamma \leqq\left[\left(\rho_{1}+\Gamma_{1}\right)\left[\rho_{2}-\left(1+\Gamma_{2}\right)\right]\right]^{1 / 2}$, we obtain

$$
r_{1}^{*+}=(\rho+1+\Gamma)^{2} \leqq\left[1+\left[\left(\rho_{1}+\Gamma_{1}\right)\left[\rho_{2}-\left(1+\Gamma_{2}\right)\right]\right]^{1 / 2}\right]^{2} .
$$


Thus it suffices to show that

$$
\left[1+\left[\left(\rho_{1}+\Gamma_{1}\right)\left[\rho_{2}-\left(1+\Gamma_{2}\right)\right]\right]^{1 / 2}\right]^{2} \leqq\left(\rho_{1}+1+\Gamma_{1}\right)\left(\rho_{2}-\Gamma_{2}\right)=r_{1}^{+},
$$

but this inequality is equivalent to the identity $\left[\left(\rho_{2}-\left(1+\Gamma_{2}\right)\right)^{1 / 2}-\left(\rho_{1}+\Gamma_{1}\right)^{1 / 2}\right]^{2} \geqq 0$. This completes the proof.

LEMMA 6.4. Let

$$
\Delta_{1}(r)=h_{1}(r)-h_{1}^{*}(r)=s_{1} / r+q_{1}+p_{1} r
$$

where

$$
\begin{aligned}
p_{1} & =c_{1}-c_{1}^{*}=1 / 2 \Gamma_{2}\left(1+\Gamma_{1}\right)+1 / 2(1+\Gamma)^{2}, \\
q_{1} & =b_{1}-b_{1}^{*}=\rho_{1} \rho_{2} /-\Gamma_{2}\left(1+\Gamma_{1}\right)-\rho^{2} /(1+\Gamma)^{2}, \\
s_{1} & =a_{1}-a_{1}^{*}=\frac{\left[\left(1+\Gamma_{1}\right)^{2}-\rho_{1}^{2}\right]\left(\Gamma_{2}^{2}-\rho_{2}^{2}\right)}{2 \Gamma_{2}\left(1+\Gamma_{1}\right)}+\frac{\left[(1+\Gamma)^{2}-\rho^{2}\right]^{2}}{2(1+\Gamma)^{2}} .
\end{aligned}
$$

If the parameters $\Gamma_{i}, \rho_{i}, \Gamma, \rho$ satisfy (6.16) and (6.18) then $p_{1} \geqq 0$ and hence

$$
\Delta_{1}(r) \geqq 0 \text { for } r_{1}^{*-} \leqq r \leqq r_{1}^{*+}
$$

provided $q_{1} \geqq 0$.

Proof. If $p_{1} \geqq 0$ and $q_{1} \geqq 0$ then (6.29) is easily verified. For if $s_{1} \geqq 0$ then $\Delta_{1}(r) \geqq 0$ for $0<r<\infty$. If $s_{1}<0$ then $\Delta_{1}(r)$ increases from $-\infty$ to $+\infty$ as $r$ passes from 0 to $\infty$ and $\Delta_{1}(r)=0$ has a simple root in $0<r<\infty$. But from Lemma 6.3(d) it follows that there are either two roots or no roots of $\Delta_{1}(r)=0$ in the interval $r_{1}^{*-} \leqq r \leqq r_{1}^{*+}$; hence there are no roots. It remains to prove that $p_{1} \geqq 0$. A simple calculation shows that $p_{1} \geqq 0$ if and only if $D_{1} \geqq 0$, where

$$
\begin{aligned}
D_{1}=- & \Gamma_{2}\left(1+\Gamma_{1}\right)\left[\left(\rho_{1}-\Gamma_{1}\right)\left(1+\Gamma_{2}\right)-\Gamma_{1}\left(\rho_{2}+1+\Gamma_{2}\right)\right]^{2} \\
-\left[\left(\rho_{1}-\Gamma_{1}\right)\left(1+\Gamma_{2}\right)-\right. & \Gamma_{1}\left(\rho_{2}+1+\Gamma_{2}\right) \\
& \left.+2 \Gamma_{1}\left(1+\Gamma_{2}\right)\left[\left(\rho_{1}-\Gamma_{1}\right)\left(\rho_{2}+1+\Gamma_{2}\right)\right]^{1 / 2}\right]^{2} .
\end{aligned}
$$

\section{Letting}

$$
\begin{aligned}
0<\alpha & =\rho_{1}-\Gamma_{1}<1, & & 0<\beta=\rho_{2}+1+\Gamma_{2}<1, \\
a & =\sqrt{ } \Gamma_{1}, & b & =\left(-\left(1+\Gamma_{2}\right)\right)^{1 / 2},
\end{aligned}
$$

we obtain by successive rearrangement

$$
\begin{aligned}
D_{1}= & \left(1+a^{2}\right)\left(1+b^{2}\right)\left(\beta a^{2}+\alpha b^{2}\right)^{2}-\left(\beta a^{2}+\alpha b^{2}+2 a^{2} b^{2}(\alpha \beta)^{1 / 2}\right)^{2} \\
= & \left(a^{2}+b^{2}+a^{2} b^{2}\right)\left(\beta a^{2}-\alpha b^{2}\right)^{2}+4 a^{2} b^{2}(\alpha \beta)^{1 / 2}\left[(\alpha \beta)^{1 / 2}\left(a^{2}+b^{2}\right)-\left(\beta a^{2}+\alpha b^{2}\right)\right] \\
= & \left(a^{2}+b^{2}+a^{2} b^{2}\right)\left(\beta^{1 / 2} a-\alpha^{1 / 2} b\right)^{2}\left(\beta^{1 / 2} a+\alpha^{1 / 2} b\right)^{2} \\
& -4 a^{2} b^{2}(\alpha \beta)^{1 / 2}\left(\beta^{1 / 2} a-\alpha^{1 / 2} b\right)^{2}+4 \alpha \beta a^{2} b^{2}(a-b)^{2} \\
= & \left(\beta^{1 / 2} a-\alpha^{1 / 2} b\right)^{2}\left[\left(a^{2}+b^{2}+a^{2} b^{2}\right)\left(\beta^{1 / 2} a-\alpha^{1 / 2} b\right)^{2}\right. \\
& \left.+4 a b(\alpha \beta)^{1 / 2}\left(a^{2}+b^{2}+a^{2} b^{2}-a b\right)\right]+4 \alpha \beta a^{2} b^{2}(a-b)^{2} .
\end{aligned}
$$


It follows that $D_{1} \geqq 0$, since $a^{2}+b^{2}+a^{2} b^{2}-a b=(a-b)^{2}+a b+a^{2} b^{2} \geqq 0$. This completes the proof of the lemma.

LEMMA 6.5. If $q_{1}$ is defined as in Lemma 6.4 and if in addition the parameters $\Gamma_{i}$, $\rho_{i}$ satisfy (6.17), then $q_{1} \geqq 0$.

Proof. An elementary calculation shows that $q_{1} \geqq 0$ if and only if $D_{2} \geqq 0$, where

$$
\begin{aligned}
D_{2}= & \rho_{1} \rho_{2}\left[2 \Gamma_{1}\left(1+\Gamma_{2}\right)\left[\left(\rho_{1}-\Gamma_{1}\right)\left(\rho_{2}+1+\Gamma_{2}\right)\right]^{1 / 2}-2 \Gamma_{1}\left(1+\Gamma_{2}\right)\right. \\
& \left.+\rho_{1}\left(1+\Gamma_{2}\right)-\rho_{2} \Gamma_{1}\right]^{2} \\
& +\Gamma_{2}\left(1+\Gamma_{1}\right)\left(\rho_{1}-\Gamma_{1}\right)\left(\rho_{2}+1+\Gamma_{2}\right)\left[\rho_{1}\left(1+\Gamma_{2}\right)-\rho_{2} \Gamma_{1}\right]^{2} .
\end{aligned}
$$

Using the notation (6.31) we obtain by operations similar to those used in the preceding lemma

$$
\begin{aligned}
D_{2}= & {\left[\left(\alpha+a^{2}\right)\left(\beta+b^{2}\right)-\alpha \beta\left(1+a^{2}\right)\left(1+b^{2}\right)\right]\left[\left(\alpha+a^{2}\right) b^{2}+\left(\beta+b^{2}\right) a^{2}\right]^{2} } \\
& +4 a^{2} b^{2}\left(\alpha+a^{2}\right)\left(\beta+b^{2}\right)\left(1-(\alpha \beta)^{1 / 2}\right)\left[a^{2} b^{2}\left(1-(\alpha \beta)^{1 / 2}\right)-\left(\alpha+a^{2}\right) b^{2}-\left(\beta+b^{2}\right) a^{2}\right] \\
= & {\left[a^{2} b^{2}(1-\alpha \beta)+a^{2} \beta(1-\alpha)+b^{2} \alpha(1-\beta)\right]\left[\left(\alpha+a^{2}\right) b^{2}-\left(\beta+b^{2}\right) a^{2}\right]^{2} } \\
& +4 a^{2} b^{2}\left(\alpha+a^{2}\right)\left(\beta+b^{2}\right)(\alpha \beta)^{1 / 2}(\sqrt{ } \beta-\sqrt{ } \alpha)\left(\sqrt{ } \beta a^{2}-\sqrt{ } \alpha b^{2}\right) .
\end{aligned}
$$

It follows from (6.3) that $(\sqrt{ } \beta-\sqrt{ } \alpha) \geqq 0$ and from (6.17) that $\left(\sqrt{ } \beta a^{2}-\sqrt{ } \alpha b^{2}\right) \geqq 0$. Thus $D_{2} \geqq 0$ and this completes the proof of the lemma.

REMARKS We conclude from Lemmas 6.4 and 6.5 that $H_{1} \subseteq H_{1}^{*}$. Since $\varphi_{1}=\varphi_{1}^{*}=0$, we obtain from Theorem 6.1 that $E_{1} \subseteq E_{1}^{*}$ and hence complete the proof of Theorem 6.2 .

It should be noted that the statement of Theorem 6.2 would not be true if condition (6.17) were removed. This can be seen by an example. Let $\Gamma_{1}=2$, $-\left(1+\Gamma_{2}\right)=4.6, \rho_{1}=2.2$ and $\rho_{2}=5$. We then obtain

$$
\begin{aligned}
A & =0.28284, & B & =1.09348, \\
\Gamma & =3.02576, & \rho & =3.30869, \\
p_{1} & =0.0010894, & q_{1} & =-0.020689, \\
r_{1}^{-} & =0.48, & r_{1}^{+} & =55.12 .
\end{aligned}
$$

Thus the hyperbolas $y=h_{1}(r), y=h_{1}^{*}(r)$ intersect at $r=4.07, r=15.64$, so that $E_{1}$ is not contained in $E_{1}^{*}$. However, it should be noted that this example does not disprove our conjecture.

As a final result we consider the concentric case where the centers $\Gamma_{1}$ and $-\left(1+\Gamma_{2}\right)$ of the circular boundaries of $V_{1}$ and $-\left(1+V_{2}\right)$ coincide.

THEOREM 6.6. Let $\left(E_{1}, E_{2}\right) \in C$, where the parameters $\Gamma_{i}, \rho_{i}$ satisfy

$$
\begin{gathered}
\Gamma_{1}=-\left(1+\Gamma_{2}\right) \quad\left(\Gamma_{1}, \Gamma_{2} \text { complex numbers }\right), \\
\left|\Gamma_{1}\right|<\rho_{1}<\left|1+\Gamma_{1}\right|, \quad\left|1+\Gamma_{2}\right|<\rho_{2}<\left|\Gamma_{2}\right| .
\end{gathered}
$$


Let $\left(E_{1}^{*}, E_{2}^{*}\right) \in C^{*}$ where the parameters $\Gamma_{i}^{*}, \rho_{i}^{*}$ satisfy

$$
\Gamma_{1}^{*}=\Gamma_{1}, \quad \Gamma_{2}^{*}=\Gamma_{2}, \quad \rho_{1}^{*}=\rho_{2}^{*}=\left(\rho_{1} \rho_{2}\right)^{1 / 2} .
$$

Then $E_{i} \subseteq E_{i}^{*}, i=1,2$.

Proof. The case with $\Gamma_{1}=-\left(1+\Gamma_{2}\right)=0$ is easily verified. Suppose that $\left|\Gamma_{1}\left(1+\Gamma_{2}\right)\right|>0$ and note that $\varphi_{i}=\varphi_{i}^{*}, i=1,2$. Thus by Theorem 6.1 it suffices to prove that

$$
\Delta_{i}(r)=h_{i}(r)-h_{i}^{*}(r)=s_{i} / r+q_{i}+p_{i} r \geqq 0, \text { for } r>0, i=1,2,
$$

where in the notation of (6.9) and Lemma 6.4

$$
s_{i}=a_{i}-a_{i}^{*}, \quad q_{i}=b_{i}-b_{i}^{*}, \quad p_{i}=c_{i}-c_{i}^{*}, \quad i=1,2 .
$$

But a simple computation shows that

$$
s_{i}=\left(\rho_{2}-\rho_{1}\right)^{2} / 2 \geqq 0, \quad p_{i}=q_{i}=0, \quad i=1,2,
$$

from which the proof follows immediately.

\section{REFERENCES}

1. J. D. Bankier and Walter Leighton, Numerical continued fractions, Amer. J. Math. 64 (1942), 653-668. MR 4, 81.

2. George Copp, Some convergence regions for a continued fraction, Dissertation, Univ. of Texas, Austin, 1950.

3. V. F. Cowling, Walter Leighton and W. J. Thron, Twin convergence regions for continued fractions, Bull. Amer. Math. Soc. 50 (1944), 351-357. MR 6, 47.

4. T. F. Glass and Walter Leighton, On the convergence of a continued fraction, Bull. Amer. Math. Soc. 49 (1943), 133-135. MR 4, 195.

5. P. Henrici and P. Pfluger, Truncation error estimates for Stieltjes' fractions, Numer. Math. 9 (1966), 120-138. MR 35 \#3856.

6. K. L. Hillam and W. J. Thron, A general convergence criterion for continued fractions $K\left(a_{n} / b_{n}\right)$, Proc. Amer. Math. Soc. 16 (1965), 1256-1262. MR 33 \#1613.

7. William B. Jones and R. I. Snell, Truncation error bounds for continued fractions, SIAM J. Numer. Anal. 6 (1969), 210-221.

8. William B. Jones and W. J. Thron, Convergence of continued fractions, Canad. J. Math. 20 (1968), 1037-1055. MR 37 \#6446.

9. L. J. Lange, On a family of twin convergence regions for continued fractions, Illinois J. Math. 10 (1966), 97-108. MR 32 \#4253.

10. L. J. Lange and W. J. Thron, A two-parameter family of best twin convergence regions for continued fractions, Math. Z. 73 (1960), 295-311. MR 22 \#6887.

11. Walter Leighton and W. J. Thron, On the convergence of continued fractions to meromorphic functions, Ann. of Math. (2) 44 (1943), 80-89. MR 4, 195.

12. Walter Leighton and $\mathrm{H}$. S. Wall, On the transformation and convergence of continued fractions, Amer. J. Math. 58 (1936), 267-281.

13. Oskar Perron, Uber zwei Kettenbrüche von H. S. Wall, Bayer. Akad. Wiss. Math.-Nat. Ki. S.-B. 1957, 1-13. MR 21 \#5104.

14. Vikramaditya Singh and W. J. Thron, $A$ family of best twin convergence regions for continued fractions, Proc. Amer. Math. Soc. 7 (1956), 277-282. MR 17, 1076. 
15. W. J. Thron, Two families of twin convergence regions for continued fractions, Duke Math. J. 10 (1943), 677-685. MR 5, 118.

16. - A family of simple convergence regions for continued fractions, Duke Math. J. 11 (1944), 779-791. MR 6, 210.

17. - Zwillingskonvergenzgebiete für Kettenbrüche $1+K\left(a_{n} / 1\right)$, deren eines die Kreisscheibe $\left|a_{2 n-1}\right| \leqq \rho^{2}$ ist, Math. Z. 70 (1958/59), 310-344. MR 21 \#4229.

18. - Convergence of sequences of linear fractional transformations and of continued fractions, J. Indian Math. Soc. 27 (1963), 103-127. MR 32 \#1331.

19. - On the convergence of the even part of certain continued fractions, Math. $Z$. 85 (1964), 268-273. MR 29 \#6215.

20. H. S. Wall, Partially bounded continued fractions, Proc. Amer. Math. Soc. 7 (1956), 1090-1093. MR 18, 635.

UNIVERSITY OF Colorado,

BOUlder, Colorado 80302 